\title{
A free energy study of the liquid-liquid phase transition of the Jagla two-scale potential ${ }^{\dagger}$
}

\author{
FRANCESCO RICCI ${ }^{\mathrm{a}, \mathrm{b}}$ and PABLO G DEBENEDETTI ${ }^{\mathrm{a}, *}$ \\ ${ }^{a}$ Department of Chemical and Biological Engineering, Princeton University, Princeton, NJ 08544, USA \\ ${ }^{b}$ Material and Analytical Sciences Department, Boehringer Ingelheim Pharmaceuticals Inc., Ridgefield, \\ CT 06877, USA \\ E-mail: pdebene@princeton.edu
}

MS received 29 January 2017; revised 27 March 2017; accepted 28 March 2017

\begin{abstract}
A fundamental understanding of pure-component liquid-liquid phase separation in network-forming fluids remains an open challenge. While considerable progress has been recently made in demonstrating the existence of such a phase transition in some models via rigorous free energy calculations, it remains unclear what aspects of a model are sufficient, necessary, and/or prohibited in order for it to exhibit a liquid-liquid phase transition (LLPT). Among the simplest models capable of producing water-like anomalies is the sphericallysymmetry two-scale Jagla potential, for which an LLPT has been identified via equation of state calculations. In this work, we perform rigorous free energy calculations to demonstrate the existence of an LLPT in the Jagla model. We also utilize finite-size scaling analysis to calculate the surface tension associated with the LLPT. In addition to the thermodynamics of the model, we investigate the relaxation times for density and bondorientational order in both liquid phases and show that, contrary to assertions in the literature, the characteristic relaxation time of bond-orientational order is not orders of magnitude slower than that of density. To the contrary, we actually identify conditions for which density is the slowly relaxing order parameter. In addition to the original parameterization of the Jagla model, we provide in the "Appendix" preliminary free energy surface calculations for select parameterizations of the generalized family of Jagla potentials spanning from the original (anomalous, water-like) Jagla model to the Lennard-Jones model. These calculations indicate that, as the parameterization moves towards the Lennard-Jones limit, the LLPT disappears within the range of parameters explored. Throughout the paper, we compare our results for the Jagla model with those found in the literature for the ST2 model of water in order to emphasize key similarities and differences between two models that exhibit pure-component liquid-liquid phase separation.
\end{abstract}

Keywords. Liquid-liquid critical point; phase transition; water; free energy calculation.

\section{Introduction}

The possible existence of a liquid-liquid phase transition (LLPT), and its associated liquid-liquid critical point (LLCP), in pure supercooled liquid water has been the subject of vigorous debate over the past two decades. ${ }^{1-17}$ While other hypotheses ${ }^{18,19}$ have been put forth, the existence of such a liquid-liquid critical point would serve to explain several of the celebrated anomalies of supercooled water, such as the decrease of density and increase of compressibility upon cooling, the increase in diffusivity upon compression, and the apparent divergences in its response functions as the liquid is cooled well below the freezing temperature. ${ }^{20}$ The LLCP scenario also serves as an intuitive explanation

\footnotetext{
*For correspondence

†Dedicated to the memory of the late Professor Charusita Chakravarty.
}

of the polyamorphism observed in glassy water, ${ }^{21,22}$ for which the high density amorphous (HDA) and low density amorphous (LDA) glassy phases are thought to be low-temperature structurally arrested manifestations of the high density liquid (HDL) and low density liquid (LDL), respectively. ${ }^{1}$ In addition to water, other network-forming fluids such as silicon ${ }^{23-28}$ and silica $^{29-31}$ have also been studied in order to explore the possible existence of an LLCP.

The proposed location of the LLCP in the aforementioned network-forming fluids is deep within the supercooled liquid region, below the homogeneous nucleation temperature (i.e., the empirical limit of liquid stability with respect to the crystalline phase) in a region referred to as 'no man's land'. ${ }^{32}$ Because of the experimental difficulties associated with studying the liquid phase at these extreme conditions, computer simulations have been utilized to determine whether a model 
single-component system is capable of demonstrating a first-order transition between two distinct metastable liquid phases.

Among the various models of network-forming fluids which have been investigated, the ST2 model of water ${ }^{33}$ has received perhaps the most attention, which is fitting because the metastable LLCP hypothesis was first put forth based on equation of state data obtained from that model. ${ }^{1}$ More recently, the debate over whether ST2 water exhibits an LLCP was rekindled by a vigorous discussion in the literature. ${ }^{3-11,13-17}$ Most of these investigations, utilizing state of the art simulations techniques and rigorous free energy calculations, come to the same conclusion: ST2 water exhibits a bona fide metastable LLPT which terminates at a metastable critical point.

While a bevy of evidence has been put forth supporting the LLCP scenario in the ST2 model of water, it has also been shown that other models of anomalous network-forming fluids, such as the SW model of silicon, ${ }^{34}$ and the closely related $\mathrm{mW}$ model of water $^{35}$ do not exhibit such behavior. ${ }^{4,6,28}$ Hence, it remains unclear what aspects of a model will allow for the existence of an LLPT. Smallenburg et al. ${ }^{36}$ made progress along these lines by developing a theoretical model of tetrahedrally-coordinated patchy particles with flexible patch orientation and adjustable 'softness' of inter-particle patch interactions. By modulating these parameters, the researchers were able to identify regions of parameter space for which an LLPT is metastable with respect to crystallization, as well as regions where it is stable with respect to crystallization down to zero temperature, thereby 'erasing no-man's land'.

Other models which have been investigated within the context of liquid-liquid phase separation are the socalled core-softened models. These pairwise isotropic potentials, originally introduced by Hemmer and Stell, ${ }^{37,38}$ contain a repulsive core coupled with a region of softening in the form of a shoulder or a ramp. This class of models is particularly appealing since it very simply introduces two characteristic length scales: a longer distance which allows for the creation of open, lower-density network structures at low pressure, and a shorter distance which is realized at higher pressures when the open network collapses to a more compact state. The notion of two inherent length scales is thought to be a key ingredient in the occurrence of an LLPT in water. ${ }^{39}$

Along this line of reasoning, Jagla ${ }^{40}$ developed a spherically symmetric core-softened linear-ramp model with a repulsive hard core and an attractive basin. With an appropriate choice of potential parameters, this model has been shown ${ }^{40-43}$ to exhibit an LLPT, terminating at a critical point which is stable ${ }^{43}$ with respect to freezing. In addition to an LLPT, the Jagla model is capable of producing many of the thermodynamic, dynamic, and structural anomalies of liquid water and other network-forming fluids, including a density anomaly, ${ }^{20}$ diffusivity anomaly, ${ }^{44}$ structural anomaly,${ }^{44}$ the anomalous behavior of thermodynamic response functions ${ }^{20}$ (i.e., isobaric heat capacity and isothermal compressibility), and a fragile-strong dynamic crossover ${ }^{45}$ upon crossing the Widom line (i.e., locus of maximum correlation length in the one phase region, emanating from the critical point). ${ }^{41-43,46}$ Moreover, the Jagla model reproduces several of the characteristics of glassy polyamorphism in water, including the formation of an HDA solid and an LDA solid, and a very-high density amorphous (VHDA) solid. ${ }^{47}$

The Jagla model is a striking example of a simple pair potential with two characteristic length scales which displays an LLPT and several of the anomalies of water without incorporating anisotropic interactions. This is in contrast to other models ${ }^{33,36}$ for which an LLPT has been discovered, wherein anisotropy is intrinsic to the potential. However, despite the commonality of an LLPT, the Jagla model does exhibit key differences with respect to the aforementioned ${ }^{33,36}$ models. Focusing in particular on the ST2 model of water, we note that the LLPT coexistence line in the pressure-temperature plane is negatively sloped, ${ }^{5}$ whereas in the Jagla model it is positively sloped. ${ }^{41}$ This is due to the fact that, in the Jagla (ST2) model, the HDL phase is of lower (higher) entropy than the LDL phase, and hence the Clapeyron equation dictates that $(d P / d T)_{\text {coex }}$ is $>0(<0)$. The Jagla potential also differs from ST2 and other water models in that, for the Jagla potential the HDL phase exhibits strong (i.e., Arrhenius) temperature-dependent dynamics and the LDL phase exhibits fragile (i.e., power law) behavior, while for the ST2 potential the HDL phase is fragile and the LDL phase is strong. ${ }^{43,46}$ It has been noted $^{48}$ that this inversion is generally correlated with the slope of the LLPT coexistence line, in that the more ordered (i.e., lower entropy) liquid is strong, while the less ordered (i.e., higher entropy) liquid is fragile.

The Jagla model's spherically symmetric potential lacks the tetrahedral-network character found in water, and hence it forms a 12-coordinated hexagonal close packed (HCP) crystal as opposed to a 4-coordinated tetrahedral crystal such as hexagonal ice or cubic ice. However, this can be accounted for by considering one Jagla particle as 'effectively' corresponding to two water molecules: one full water molecule plus $1 / 4$ of each of its four nearest neighbors. ${ }^{49}$ Indeed, operating under this assumption, researchers were able to assign physical units to the Jagla potential in order to facilitate comparison with other water models, and experiments. ${ }^{47,49}$ 
One issue of implementation regarding the Jagla potential is that it is not everywhere differentiable and hence gives rise to discontinuous forces. This is an issue in so far as it hinders the ability to study the potential with conventional molecular dynamics. Several researchers ${ }^{43,46,47,50}$ have accordingly implemented discrete molecular dynamics simulations in which the continuous Jagla potential is approximated by a series of step functions. More recently, Abraham et al., ${ }^{48}$ developed a smooth version of the Jagla potential, which they term the Fermi-Jagla potential. The Fermi-Jagla potential qualitatively maintains the important waterlike anomalies of the original potential, including the LLPT, with the exception that the liquid-liquid coexistence line has a slightly negative slope in the $P-T$ plane.

Beyond the original Jagla potential, ${ }^{40}$ one may consider a generalized family of Jagla potentials by tuning parameters such as the depth of the potential minimum and the distance to the potential minimum with respect to the hard-core distance. It has previously been illustrated for non-attractive ramp potentials that the ratio of the two characteristic length scales must be in the vicinity of $\sim 0.57$ in order to most closely reproduce, in a qualitative sense, the anomalies of water. ${ }^{51}$ This is thought to be related to the fact that the ratio of radial distances to the first and second peaks of the oxygenoxygen pair correlation function in water is $\sim 0.6$, while the ratio for simple liquids is $\sim 0.5$. The original Jagla model has a ratio of length scales $\sim 0.58$, which is very close to the value for real water.

In a very interesting study, Gibson and Wilding ${ }^{42}$ investigated the generalized family of Jagla potentials for which they varied both the depth of, and the radial distance to the potential minimum, so as to interpolate between the original Jagla potential and a ramp-based approximation to the Lennard-Jones potential. As the researchers varied the potential from the original Jagla parameterization towards the Lennard-Jones limit, they observed a decrease in the liquid-liquid critical temperature, and an increase in the temperature at which the liquid spontaneously crystallized, leading to a condition where the LLCP (and thereby the entire LLPT) was unstable with respect to crystallization. We note that the "freezing point"as defined by these authors appears to be the temperature (for a given pressure) at which the liquid becomes unstable and the system spontaneously crystallizes, not the true equilibrium freezing/melting point. The equilibrium freezing/melting line for the Jagla potential was calculated by $\mathrm{Xu}$ et al.,${ }^{43}$ and the intersection of the freezing line and LLPT coexistence line is only slightly below the critical temperature. Irrespective of this issue, the authors observed that upon moving further towards the Lennard-Jones limit, the slope of the liquid-liquid coexistence line changed from positive to negative. Just beyond this crossover to a negatively sloped coexistence line, the increasing rate of spontaneous crystallization at the relevant conditions made determination of the LLCP prohibitive. Since the Lennard-Jones fluid does not exhibit an LLPT, it stands to reason that there must be some point between the Jagla potential limit and the Lennard-Jones approximation limit at which the LLPT disappears altogether; however, the study of Gibson and Wilding ${ }^{42}$ was unable to definitively determine for what parameterization this occurs.

More recently, Luo et al., ${ }^{50}$ performed an analogous study in which they investigated the LLPT by examining the behavior of the Widom line while modifying the Jagla potential. However, their modification was slightly different in that they chose to maintain a constant potential minimum depth while continuously transforming the minimum's radial distance with respect to the hard-core distance. As with the study of Gibson and Wilding, ${ }^{42}$ they observed that decreasing the radial distance of the potential-minimum caused the LLCP to retreat to lower temperatures, while the slope of the liquid-liquid coexistence line correspondingly decreased, and eventually became slightly negative. The researchers also reported that ${ }^{50}$ at precisely the same ratio of length scales (hard-core to potential minimum radial distances) as in the Gibson and Wilding study, locating the LLCP became impossible due to prohibitively rapid crystallization in their simulations.

While a bevy of equation of state calculations have been put forth illustrating an LLPT for the Jagla potential, there has not yet been a rigorous free energy surface study of the type performed for the ST2 model. Given the fact that equation of state calculations alone may lead to the false impression of an LLPT, ${ }^{28}$ we will, for the first time, perform explicit free energy surface calculations to explore the existence or lack thereof of an LLPT in the Jagla model. We utilize free energy techniques, coupled with finite-size scaling analysis, for the calculation of liquid-liquid surface tension in the Jagla potential. We also investigate bond-orientational and translational order in the context of the LLPT. In addition to the thermodynamics of the model, we investigate the oft-discussed relaxation times of density and bond-orientational order for both the HDL and LDL phases, and discuss the implications of our results with respect to similar calculations performed for the ST2 model. Beyond the original parameterization of the Jagla model, we also provide in the "Appendix" a preliminary investigation of free energy surfaces for select parameterizations of the generalized family of Jagla potentials which range from the original (anomalous) Jagla model towards the limit of the 
(simple) Lennard-Jones model. Our results indicate that, as the parameterization moves towards the limit of the Lennard-Jones model, the LLPT seems to disappear within the range of parameters explored. Throughout the paper, we compare our results for the Jagla model with those found in the literature for the ST2 model of water in order to emphasize key similarities and differences between two models which exhibit purecomponent liquid-liquid phase separation.

The paper is organized as follows. In Section 2, we describe the model, simulation methods, and relevant order parameters. In Section 3, the results of our study are presented and discussed. Finally, in Section 4, concluding remarks are provided, along with suggestions for future work.

\section{Models and numerical methods}

\subsection{Model system}

The Jagla attractive two-scale spherically-symmetrical ramp potential ${ }^{40}$ is a pairwise potential in which the energy of interaction, $v$, between two particles separated by some distance, $r$, is the sum of an attractive term and a repulsive term, as given by Equation (1):

$$
v(r)=v_{A}(r)+v_{R}(r)
$$

where, $v_{A}(r)$ is the attractive part of the potential, and $v_{R}(r)$ is the repulsive part of the potential, each defined as:

$$
\begin{aligned}
& v_{R}(r)= \begin{cases}\infty & ; \quad r<r_{0} \\
\varepsilon_{0}\left(r_{1}-r\right) /\left(r_{1}-r_{0}\right) ; & r_{0} \leq r \leq r_{1} \\
0 & ; \quad r>r_{1}\end{cases} \\
& v_{A}(r)= \begin{cases}-\eta\left(r_{2}-r\right) /\left(r_{2}-r_{0}\right) & ; \quad r \leq r_{2} \\
0 & ; \quad r>r_{2}\end{cases}
\end{aligned}
$$

where, $r_{0}$ is the hard-core distance, $r_{1}$ is the intermediate distance which defines the location of the potential minimum, and $r_{2}$ is the distance beyond which the potential is identically zero. These distances are subject to the constraint $r_{0}<r_{1}<r_{2}$. The energetic parameters are $\varepsilon_{0}$ and $\eta$, subject to $\eta>0$. For this potential, we adopt the parameterization originally proposed by Jagla ${ }^{40}: r_{1}=1.72 r_{0}, r_{2}=3.0 r_{0}$, and $\eta=0.31 \varepsilon_{0}$. Unless otherwise specified, all quantities are expressed in reduced units, with the basis length scale set to $r_{0}$, and the basis energy scale set to $\varepsilon_{0}$. Hence, temperature is expressed in units of $\varepsilon_{0} / k_{B}$, where $k_{B}$ is Boltzmann's constant, pressure is expressed in units of $\varepsilon_{0} / r_{0}^{3}$, and density is expressed in units of $r_{0}^{-3}$.

We note that other researchers ${ }^{43,46,47,52}$ have employed an alternative (but equivalent) functional form with energy scale " $U_{0}$ ". In this alternative functional form, $U_{0}$ represents the magnitude of the energy minimum of the attractive basin, $v\left(r_{1}\right)$, while $U_{R}$ represents the energy at the top of the repulsive ramp, $v\left(r_{0}\right)$ (refer to Figure 1 for a graphical representation of the Jagla potential). Some studies ${ }^{43,46,47}$

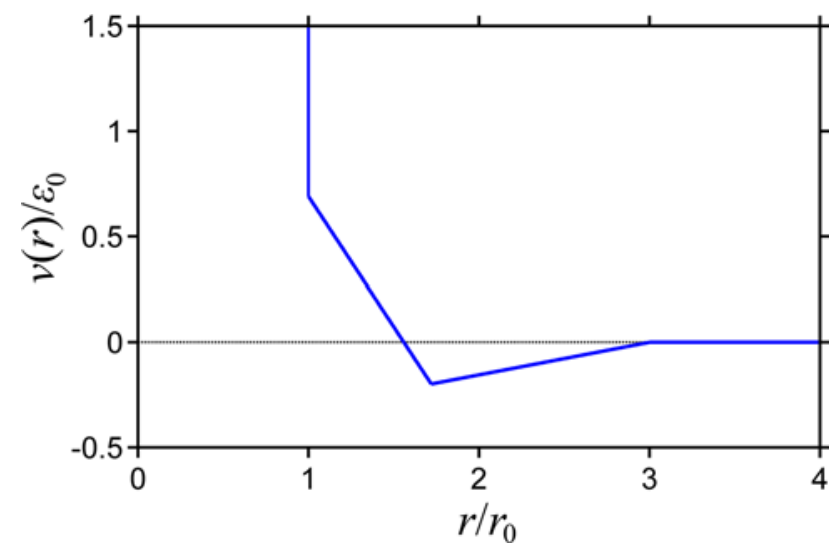

Figure 1. Graphical illustration of the attractive two-scale spherically-symmetrical ramp potential of Jagla. ${ }^{40}$ The parameterization is specified by $r_{1}=1.72 r_{0}, r_{2}=3.0 r_{0}$, and $\eta=0.31 \varepsilon_{0}$. The $v(r) / \varepsilon_{0}=0$ line is provided to emphasize the potential's shallow attractive well.

employ a ratio $U_{R} / U_{0}=3.5$, while other studies ${ }^{53,54}$ employ $U_{R} / U_{0}=3.56$. On the other hand, if one uses the energy parameterization of the original Jagla potential, ${ }^{40}$ the ratio is $U_{R} / U_{0}=3.48$. This difference may seem miniscule, but it has a non-negligible effect on the certain aspects of the model, including the location of the liquid-liquid critical point, as discussed by Xu et al. ${ }^{53}$ To facilitate a comparison between the original Jagla energy parameterization which we study, and the alternative energy parameterizations, we set equal the values of the potential minimum for each functional form. After simple algebra, one can calculate the relation $U_{0}=0.1984 \varepsilon_{0}$. With this conversion factor, we calculate that the difference in reported ${ }^{41,53}$ critical temperatures between the slightly different energy parameterizations $U_{R} / U_{0}=3.56$ vs. 3.48 , is roughly $0.0016 \varepsilon_{0} / k_{B}$, or $2 \%$ of the critical temperature calculated ${ }^{41}$ for the original Jagla parameterization. We also point out that the vast majority of studies which employ this alternative energy scale also employ the discrete molecular dynamics technique, in which the Jagla potential is approximated by a series of step functions. Hence, slight discrepancies in results may also be expected due to the implementation of this approximation.

Applying the original parameterization, ${ }^{40}$ the Jagla potential is illustrated in Figure 1. Upon examination, the two inherent length scales are immediately obvious: the attractive basin at $r_{1}$, and the hard-core at $r_{0}$.

With the pair-interaction potential defined by Equations (1)-(3), it is a simple matter to calculate the total internal energy, $U$, of a system of $N$ particles:

$$
U(1, \ldots, N)=\sum_{i=1}^{N-1} \sum_{j=i+1}^{N} v\left(r_{i j}\right)
$$

Where, $r_{i j}$ is the distance $r$ between particles $i$ and $j$.

In addition to the aforementioned original parameterization of the Jagla potential, ${ }^{40}$ we provide in the "Appendix" a preliminary investigation of the generalized family of Jagla 
potentials by varying the length scales $r_{1}$ and $r_{2}$, and the depth of the potential-minimum located at $r_{1}$, as previously explored by Gibson and Wilding. ${ }^{42}$ In their work, ${ }^{42}$ these authors examined a range of parameters spanning between the original Jagla potential and a ramp-based approximation to the 6-12 Lennard Jones potential. To facilitate the manner in which Gibson and Wilding varied the energy and length parameters of the Jagla potential, they rearranged the form given in Equations. (1)-(3), and expressed it in the following equivalent way:

$$
v(r)=\left\{\begin{array}{lll}
\infty & ; \quad r<r_{0} \\
\frac{\varepsilon_{0}\left(r_{0}-r\right)(D+\omega)}{r_{1}-r_{0}}+\varepsilon_{0} \omega & ; \quad r_{0} \leq r<r_{1} \\
\varepsilon_{0} D\left(r_{2}-r\right) /\left(r_{1}-r_{2}\right) ; & r_{1} \leq r<r_{2} \\
0 & ; \quad r \geq r_{2}
\end{array}\right.
$$

where, the new terms $D$ and $\omega$ are simply the following regroupings:

$$
\begin{aligned}
D & =\frac{\eta\left(r_{2}-r_{1}\right)}{\varepsilon_{0}\left(r_{2}-r_{0}\right)} \\
\omega & =1-\frac{\eta}{\varepsilon_{0}}
\end{aligned}
$$

\subsection{Simulation methods and order parameters}

In the present study, we performed Monte Carlo simulations in multiple ensembles. Standard single-particle move Metropolis Monte Carlo (MC) simulations were performed in the canonical (NVT) and isothermal-isobaric (NPT) ensembles using an in-house code developed for the Jagla potential. The in-house NPT MC code was also employed to perform umbrella sampling simulations. Periodic boundary conditions were employed in all simulations. Unless otherwise noted, a cubic simulation box geometry was employed.

For all NVT MC calculations, the pressure was calculated using a trial volume perturbation method, as described in the literature. ${ }^{55}$ For NVT simulations, a MC 'sweep' is defined as $N$ trial single-particle displacements, where $N$ is the total number of particles. During the equilibration period of the NVT simulations, the maximum particle displacement was adjusted to give an acceptance ratio of approximately 20$30 \%$.

For all NPT MC calculations the ratio of trial singleparticle displacements to trial volume changes was $N: 1$, where $N$ is the number of Jagla particles in the system. For NPT simulations, we define a MC sweep as either $N$ attempted single-particle displacements, or one attempted volume change. As with the maximum displacement distance, the maximum logarithmic volume changes were adjusted to give an acceptance ratio of approximately 20-30\%.

The umbrella sampling MC calculations were performed in the NPT ensemble, using the commonly-employed ${ }^{4-6,8}$ harmonic biasing potential:

$$
U_{\text {bias }}=k_{\rho}\left(\rho-\rho^{*}\right)^{2}+k_{Q_{6}}\left(Q_{6}-Q_{6}^{*}\right)^{2}
$$

where, $\rho$ is the density of the system and $Q_{6}$ is the customary bond-orientational order parameter, ${ }^{56}$ which is used to distinguish crystalline and liquid states. The parameters $\rho^{*}$ and $Q_{6}^{*}$ are the specified target values of density and bond-orientational order parameter in each umbrella sampling window. The parameters $k_{\rho}$ and $k_{Q_{6}}$ are constants which may be tuned so that there is sufficient histogram overlap between adjacent windows. We typically adopt the values $k_{\rho}=1,500 \varepsilon_{0} r_{0}^{6}, k_{Q_{6}}=1,000 \varepsilon_{0}$. Spacing between umbrella windows (i.e., between target values) was typically $0.025 r_{0}^{-3}$ in $\rho^{*}$, and 0.02 in $Q_{6}^{*}$, although finer spacing was used as necessary to ensure adequate histogram sampling.

The order parameter $Q_{6}$ is a function of the system's averaged spherical harmonic components, ${ }^{56}$ defined generally as $Q_{l}$ by Equations (9)-(11):

$$
q_{l, m}(i)=\frac{1}{N_{1}} \sum_{j \in n n(i)}^{N_{1}} Y_{l}^{m}\left(\phi_{i j}, \theta_{i j}\right), \quad-l \leq m \leq l
$$

where, $Y_{l}^{m}\left(\phi_{i j}, \theta_{i j}\right)$ is the $l, m$ spherical harmonic function of the angular coordinates of the vector joining particles $i$ and $j$, and $N_{1}$ is the number of nearest-neighbors in the first coordination shell. We take $N_{1}=12$ for the 12 -fold coordinated Jagla system, which forms an HCP crystal. This term is then summed over all particles to calculate $Q_{l, m}$ :

$$
Q_{l, m}=\sum_{i=1}^{N} q_{l, m}(i)
$$

where, $N$ is the system size. Finally, we obtain the global order parameter $Q_{l}$ :

$$
Q_{l}=\frac{1}{N}\left(\sum_{m=-l}^{l} Q_{l, m} \hat{Q}_{l, m}\right)^{1 / 2}
$$

where, we use the circumflex () to denote the complex conjugate. The parameter $Q_{6}$ is a scalar quantity, whose value is 0.485 for a perfect HCP lattice. ${ }^{57}$ For amorphous systems, $Q_{6}$ has a small, nonzero value for finite-sized systems (i.e., $\sim 0.02-0.05$ for the system size range studied), which vanishes as $N^{-1 / 2}$ with increasing system size.

The translational order parameter, ${ }^{58} \tilde{t}$, measures the proclivity of pairs of particles to adopt preferential separations. This quantity, given by Equation (12), vanishes for an ideal gas and is large for a crystal.

$$
\tilde{t}=\frac{1}{r_{c}} \int_{0}^{r_{c}}\left|g^{(2)}(r)-1\right| d r
$$

where, $g^{(2)}(r)$ is the radial distribution function, and $r_{c}$ is the cutoff distance, which we choose as half of the simulation box length, $L / 2$.

The entropy of a monatomic, homogeneous, isotropic fluid can be expressed an expansion in multiparticle correlation functions, expressed generally as: ${ }^{59,60}$

$$
S=S^{i g}+\sum_{n=2}^{N} S^{(n)}
$$


where, $S$ is the entropy, $S^{i g}$ is the entropy of an ideal gas at the same temperature and density as the system of interest, and $S^{(n)}$ is the $n$-body correlation contribution to the entropy. Note that all entropic quantities are expressed on a per-particle basis in units of $k_{B}$. We choose to truncate at the two-body correlations, yielding $S=S^{i g}+S^{(2)}$. The ideal gas contribution to the entropy is given by:

$$
S^{i g}=\frac{5}{2}+\ln \left(\rho \Lambda^{3}\right)
$$

where, $\Lambda$ is the thermal de Broglie wavelength. In order to express the thermal de Broglie wavelength in reduced units, we chose to incorporate the conversion factors put forth by $\mathrm{Xu}$ et $a l .{ }^{47}$ however, this choice is not particularly important, since the thermal de Broglie wavelength is constant for a given temperature, and we are only interested in computing the change in entropy between state points at isothermal conditions. The two-body contribution to the entropy, $S^{(2)}$, is given by: ${ }^{59,60}$

$$
S^{(2)}=-2 \pi \rho \int_{0}^{\infty}\left[g^{(2)}(r) \ln g^{(2)}(r)-g^{(2)}(r)+1\right] r^{2} d r
$$

\subsection{Free energy surfaces}

From our NPT umbrella sampling simulations, we calculated two-dimensional Gibbs free energy surfaces, $F\left(\rho, Q_{6}\right)$, via the weighted histogram analysis method ${ }^{61}$ (WHAM). The free energy at a particular combination of $\rho$ and $Q_{6}$ is related to the joint probability distribution of the order parameters, $P\left(\rho, Q_{6}\right)$, by the relation:

$$
F\left(\rho, Q_{6}\right)=-k_{B} T \ln P\left(\rho, Q_{6}\right)+\text { const } .
$$

where const. is an irrelevant constant, since we are only interested in relative free energies at a given temperature. Error estimates for the free energy surfaces were calculated by the bootstrapping method, ${ }^{62}$ generating 200 independent surfaces in order to calculate the standard deviation.

A free energy surface, calculated at temperature $T$ and pressure $P$, can be reweighted in order to approximately determine the free energy surface at the same temperature and some new pressure $P+\Delta P$, provided that $|\Delta P|$ is not too large. This reweighting is carried out according to the following expression:

$$
F\left(\rho, Q_{6} ; T, P+\Delta P\right)=F\left(\rho, Q_{6} ; T, P\right)+N \Delta P / \rho
$$

Note that Equation (17) is simply derived from the thermodynamic relation $(\partial G / \partial P)_{T}=V$, replacing the Gibbs free energy, $G$, with the Landau free energy, $F$ (a more detailed discussion of Landau free energies, and how they relate to the free energy of phases is provided in "Appendix A.4") approximating the infinitesimal change, $\partial$, as a finite change, $\Delta$, and noting that $V=N / \rho$.

In order to determine the free energy as a function of density alone, the two-dimensional surface may be contracted to obtain a free energy profile via:

$$
F(\rho)=-k_{B} T \ln \left(\int \exp \left[-\beta F\left(\rho, Q_{6}\right)\right] d Q_{6}\right)
$$

where the integration is performed over the $Q_{6}$ range of interest, typically chosen based on the specific topology of the two-dimensional free energy surface. A corresponding expression for the free energy as a function of $Q_{6}$ alone, $F\left(Q_{6}\right)$, is trivially derived by instead integrating along the density dimension.

The run durations for the free energy surface simulations were based on the autocorrelation functions of $\rho$ and $Q_{6}$, defined generally as: 63

$$
C_{\xi}(t)=\frac{\langle(\xi(t)-\langle\xi\rangle)(\xi(0)-\langle\xi\rangle)\rangle}{\left\langle\xi^{2}\right\rangle-\langle\xi\rangle^{2}}
$$

where $\xi$ represents the order parameter ( $\rho$ or $Q_{6}$ ). The "characteristic" relaxation time for each of the order parameters, $\tau_{\xi}$, was defined as the integrated autocorrelation time, given by the following relation: 64

$$
\tau_{\xi}=\sum_{k=0}^{K_{f}}\left(1-\frac{k}{K_{f}}\right) t_{\text {sample }} C_{\xi}\left(k t_{\text {sample }}\right)
$$

where, $t_{\text {sample }}$ is the time interval (in units of MC sweeps) between the data points comprising the autocorrelaton function, $k$ is the index of the integer number of data points making up the autocorrelation function $\left(k=t / t_{\text {sample }}\right)$ and $K_{f}$ is the final data point of the autocorrelation function incorporated into the integration (i.e., we perform the integration until time $t_{f}=t_{\text {sample }} K_{f}$ ). We choose to perform the integration until the autocorrelation function first crosses zero. Equilibration and production periods were both typically performed for at least $\sim O\left(10^{3}-10^{4}\right)$ times the larger of the two integrated autocorrelation times (i.e., $\tau_{\rho}$ vs. $\tau_{Q_{6}}$ ) at the given state point. Additional details regarding equilibration and production durations are provided as needed insubsequent sections.

\section{Results and Discussion}

\subsection{Thermodynamics and free energy surfaces}

In order to locate the LLPT in this model, we first perform equation of state calculations in the NVT and NPT ensembles, as performed by several others. ${ }^{40,41,43}$ In particular, we examine the behavior of isotherms to check for signatures of a first-order fluid-fluid phase transition. In the NVT ensemble, an isotherm which traverses such a phase transition exhibits a non-monotonicity in the $P-V$ plane referred to as a "van der Waals (vdW) loop". Though, we note that this loop is only in loose analogy with that exhibited by cubic equations of state such as the van der Waals equation. In finite-sized simulations, a vdW loop is formed due to the relatively large free energy cost associated with forming an interface between phases, and hence the loop becomes progressively flatter as the system size is increased, coinciding 
(a)

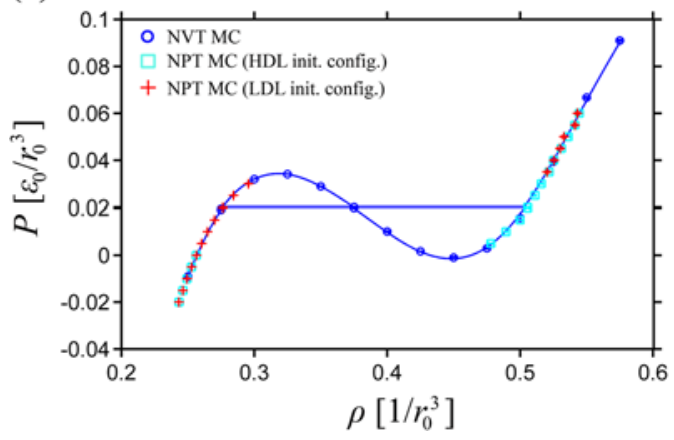

(b)

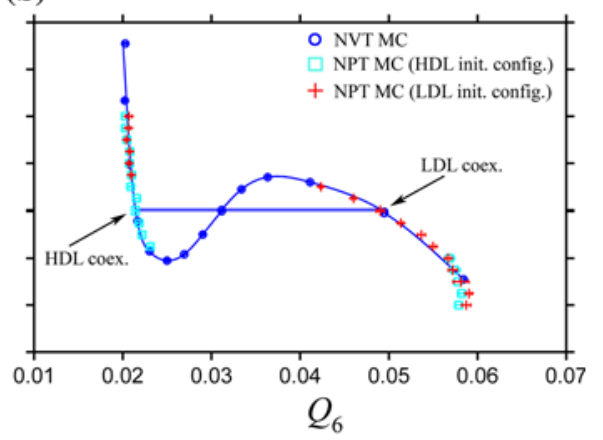

Figure 2. Equation of state analysis of the LLPT of the Jagla potential for $T=0.06 \varepsilon_{0} / k_{B}, N=300$ particles. (a) pressure vs. density, (b) pressure vs. $Q_{6}$. Calculations performed via NVT Monte Carlo (blue circles), and NPT Monte Carlo initiated with both HDL (cyan squares) and LDL (red crosses) configurations. The NVT data was fit with a smoothing spline, and the coexistence pressure (blue horizontal tie line) was determined via Maxwell equal-area construction in the $P-V$ plane. Note the hysteresis evident in the NPT simulations. Error bars are calculated from block averaging of the data.

with the coexistence pressure (i.e., the tie line) in the thermodynamic limit. However, this analogy is rather useful in determining phase equilibrium properties, as we will verify below with our free energy calculations.

An example vdW loop, calculated via NVT Monte Carlo, is illustrated in Figure 2(a), at temperature $T=$ $0.06 \varepsilon_{0} / k_{B}$. Based on the reported ${ }^{41}$ liquid-liquid critical temperature, $T_{C}^{L L}=0.076(2) \varepsilon_{0} / k_{B}$, the reduced temperature $\left(T_{r}=T / T_{C}^{L L}\right)$ at this condition is 0.79 . Note the term "reduced temperature" is not to be confused with "reduced units" as described in Section 2.1, which simply means that all quantities are normalized by basis length and energy scales.

The points on the isotherm for which $(\partial P / \partial \rho)_{T}=0$ are the intrinsic limits of stability for the liquid phases, known as spinodal points. The coexistence pressure (binodal pressure), $P_{\text {binodal }}$ between the two liquids at the given temperature is determined by performing a Maxwell equal-area construction over the vdW loop in the $P-V$ plane. In order to perform the Maxwell construction and determine the spinodal pressures, we fit the isotherm data with a smoothing spline. For $T=$ $0.06 \varepsilon_{0} / k_{B}, P_{\text {binodal }}$ is approximately $0.02 \varepsilon_{0} / r_{0}^{3}$, as illustrated by the horizontal tie line in Figure 2.

We also search for signatures of a fluid-fluid phase transition by performing simulations in the NPT ensemble. Referring to Figure 2(a), we perform two sets of NPT Monte Carlo simulations for the temperature of interest, with each set comprised of the same collection of state points. One set is initiated from an LDL configuration, while the other set is initiated from an HDL configuration. Both initial configurations were initially equilibrated at the temperature of interest (in the case of Figure 2, $\left.T=0.06 \varepsilon_{0} / k_{B}\right)$. After re-equilibrating the simulations in each set to appropriate pressure, we observe that the simulations in the metastable region $\left(P_{\text {spinodal }}^{H D L}<P<P_{\text {spinodal }}^{L D L}\right.$ ) can persist in their initiated phase for extended periods of time. However, as the respective spinodals are approached, there comes a point beyond which the simulations spontaneously change to the stable phase before equilibration is possible. This type of hysteresis in the NPT ensemble is another clear signature of a phase transition.

Referring to Figure 2(b), we also note that in the Jagla potential the average $Q_{6}$ shows very interesting behavior. At coexistence, the HDL and LDL phases for $N=$ 300 have average $Q_{6}$ values of approximately 0.02 and 0.05 , respectively, with the characteristic loop behavior connecting the two. Hence, while $Q_{6}$ values for amorphous phases are indeed system-size dependent and tend towards zero as $N \rightarrow \infty$, the bond-orientational order parameter is able to distinguish between the HDL and LDL phases in the Jagla potential. We also note that, in spite of the fact that the HDL phase has a lower entropy than the LDL phase at coexistence ${ }^{41}$ (implying that the HDL is the more structurally-ordered liquid phase for the Jagla potential), the HDL phase has lower bondorientational order than the LDL phase. We found this result interesting, and therefore decided to examine the translational order, $\tilde{t}$, of both liquid phases at coexistence as shown in Figure 3(a). Referring to this figure we see that, at coexistence, the HDL has a higher degree of translational order than the LDL phase. The behavior of the translational order appears to be clearly reflected in the entropy (truncated at the two-body contribution) as shown in Figure 3(b). As previously reported, ${ }^{41}$ we observe that the HDL phase is indeed the lower-entropy phase at coexistence. 
(a)

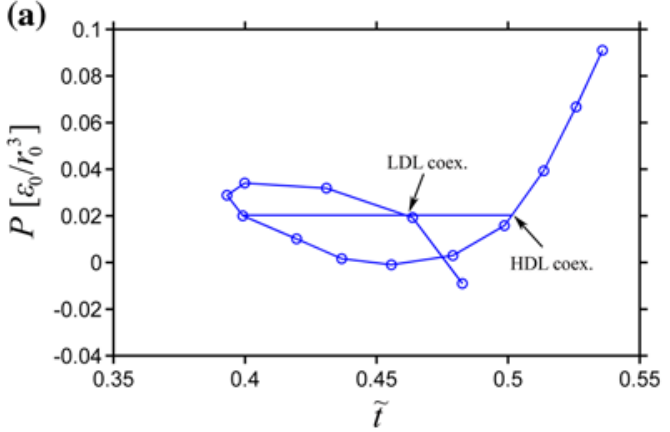

(b)

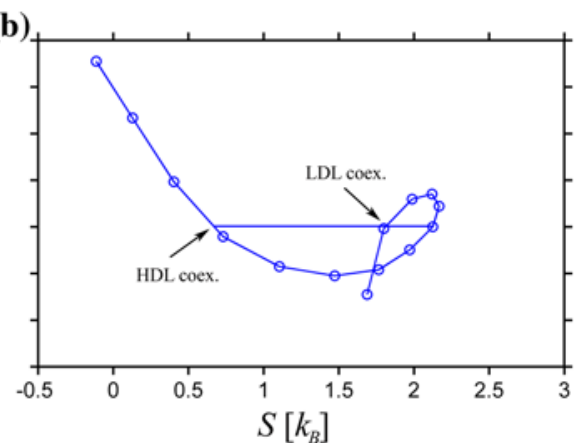

Figure 3. Plots of (a) pressure vs. $\tilde{t}$ and (b) pressure vs. entropy at $T=0.06 \varepsilon_{0} / k_{B}$, $N=300$ particles. Calculations performed via NVT Monte Carlo. The coexistence pressure (blue horizontal tie line) was determined via Maxwell equal-area construction in Figure 2(a). The entropy was calculated up to the two-body contributions via the combination of Equations (14) and (15). At coexistence, the HDL phase has higher translational order than the LDL phase, and also has lower entropy.

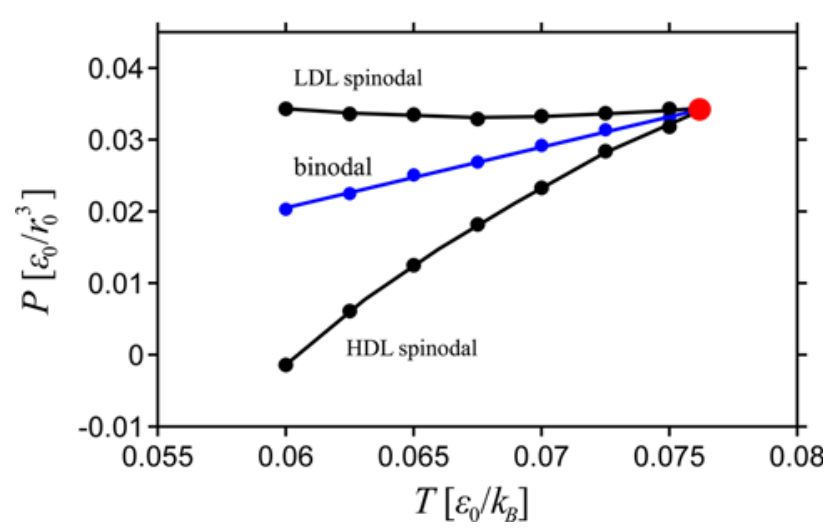

Figure 4. Pressure-Temperature diagram of the Jagla potential, $N=300$ particles. The binodal curve (blue circles) and spinodal curves (black circles) were calculated as illustrated in Figure 2(a). The red circle is the reported LLCP of Wilding and Magee. ${ }^{41}$ The lines serve as guides to the eye.

By performing a series of Maxwell equal-area constructions at various subcritical temperatures $(T<$ $T_{C}^{L L}$ ), we are able to trace the binodal and spinodal curves for the LLPT. These results, previously demonstrated elsewhere ${ }^{43}$ for a discretized step function approximation to the Jagla potential, are shown in Figure 4. As observed previously, the liquid-liquid coexistence locus is both positively sloped and approximately linear in the $P-T$ plane over the range of temperatures explored. ${ }^{43}$ If one assumes linearity over the entire temperature range and fits a line to the coexistence points in Figure 4, we find that $(d P / d T)_{\text {coex }}$ is a constant approximately equal to $0.86 k_{B} / r_{0}^{3}$. According to the Clapeyron equation, this derivative is equal to the ratio of entropy and volume changes between the HDL and LDL phases. For $T=0.06 \varepsilon_{0} / k_{B}$ we can obtain the coexistence entropies for each phase from Figure 3(b), and the coexistence volumes from Figure 2(a), calculating that $(d P / d T)_{\text {coex }}=\Delta S / \Delta V=0.7 k_{B} / r_{0}^{3}$, which is a $14 \%$ discrepancy. This discrepancy is likely primarily due to the fact that the entropy in Figure 3(b) is truncated at the two-body contributions.

Once the binodal and spinodals were determined, we proceeded to calculate free energy surfaces as a function of $\rho$ and $Q_{6}$. An example of this provided in Figure 5 , which is for the same temperature as Figure 2 $\left(T=0.06 \varepsilon_{0} / k_{B}\right)$. These free energy surfaces have the same qualitative features as free energy surfaces previously calculated for the ST2 model. ${ }^{5,8,9}$ Namely, there are two low- $Q_{6}$ basins of differing density, representing the HDL and LDL phases. At the predicted coexistence pressure, the basins are of equal depth, and at the predicted spinodal pressures, one of the basins disappears. As observed for the ST2 model, the LDL basin exhibits a more gradual (i.e., less steep) variation in the $Q_{6}$ direction than the HDL basin. ${ }^{8,9}$ Note that since the temperature $0.06 \varepsilon_{0} / k_{B}$ is well below the liquid-liquid critical temperature $\left(T_{r}=0.79\right)$, the free energy barrier separating the HDL and LDL basins $\left(\approx 47 k_{B} T\right)$ is significantly larger than the barriers typically observed in free energy studies of ST2 for comparable system sizes (i.e., 3-4 $\left.\left.k_{B} T\right)\right)^{5,9}$ This is because free energy studies of ST2 have generally been performed at much higher reduced temperatures (i.e., $T_{r}=0.96-0.97^{5,8,9}$ ) as calculations are hindered by significantly slower relaxation dynamics and increased computational costs due to the relative complexity of the potential, making lower temperatures difficult to investigate. An example free energy surface at a reduced temperature $T_{r}=0.97$ is provided in Figure 6, where the barrier separating the liquid basis at coexistence is approximately $2 k_{B} T$. We also take this opportunity to point out that at the lowest temperatures studied we found it difficult to sample the 
(a) $P=-0.01 \varepsilon_{0} / r^{3}$

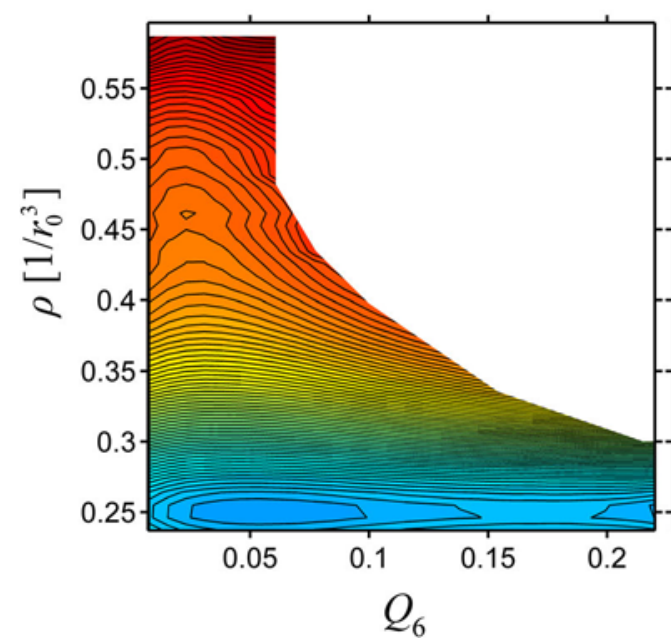

(b) $P=0.02 \varepsilon_{0} / r^{3}$

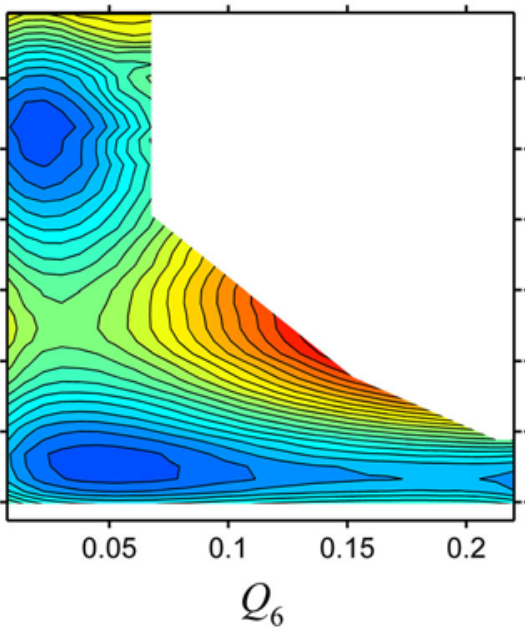

(c) $P=0.035 \varepsilon_{0} / r^{3}$

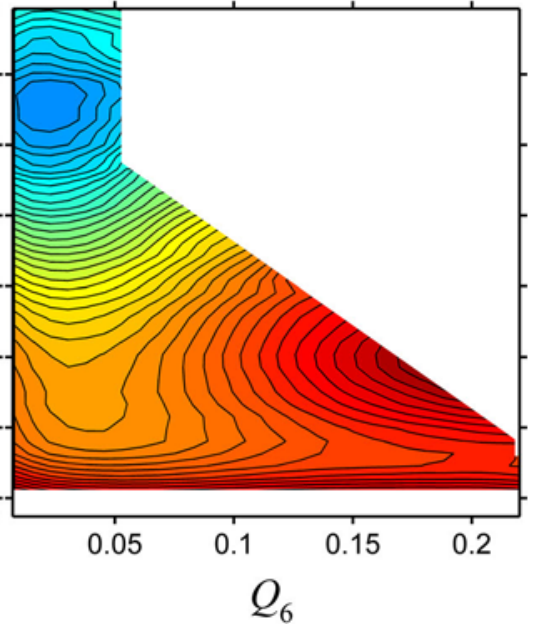

Figure 5. Free energy surfaces for the Jagla potential, at $T=0.06 \varepsilon_{0} / k_{B}, N=300$ particles, and pressures of (a) $-0.01 \varepsilon_{0} / r_{0}^{3}$, (b) $0.02 \varepsilon_{0} / r_{0}^{3}$, and (c) $0.035 \varepsilon_{0} / r_{0}^{3}$. Agreement between the binodal and spinodal pressures predicted in Figure 2 and the free energy surfaces is excellent. Contour spacing is $5 k_{B} T$, and the statistical uncertainties are less than, or on the order of $1-2 k_{B} T$ for most of the bins.

(a) $P=0.03 \varepsilon_{0} / r^{3}$

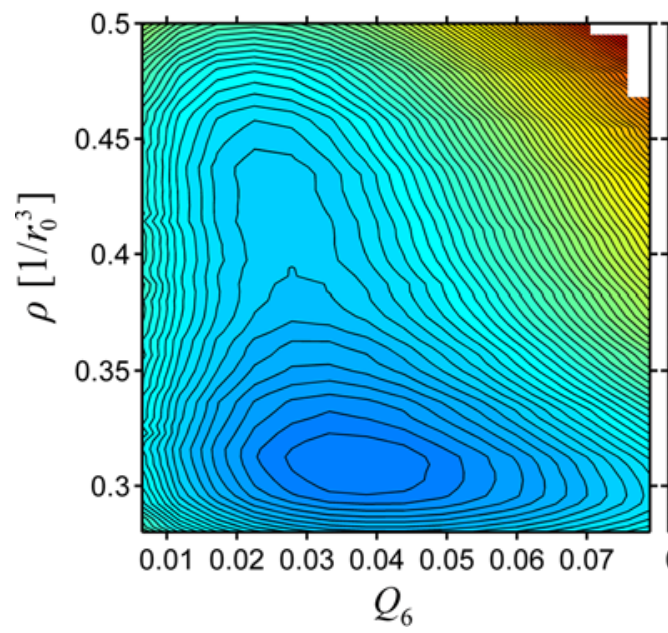

(b) $P=0.0322 \varepsilon_{0} / r^{3}$

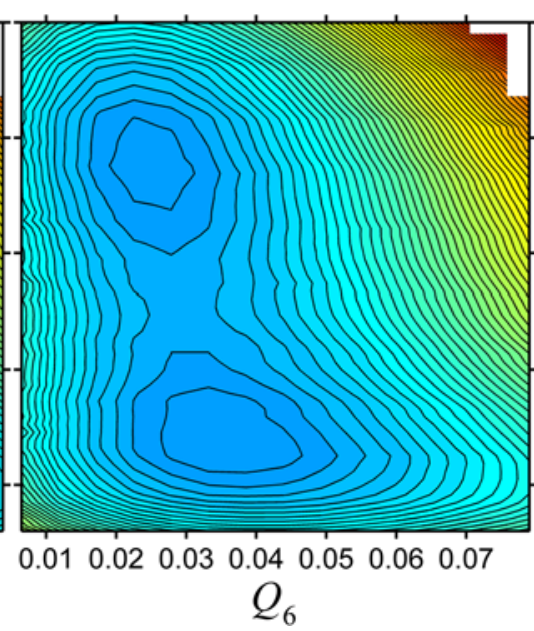

(c) $P=0.0343 \varepsilon_{0} / r^{3}$

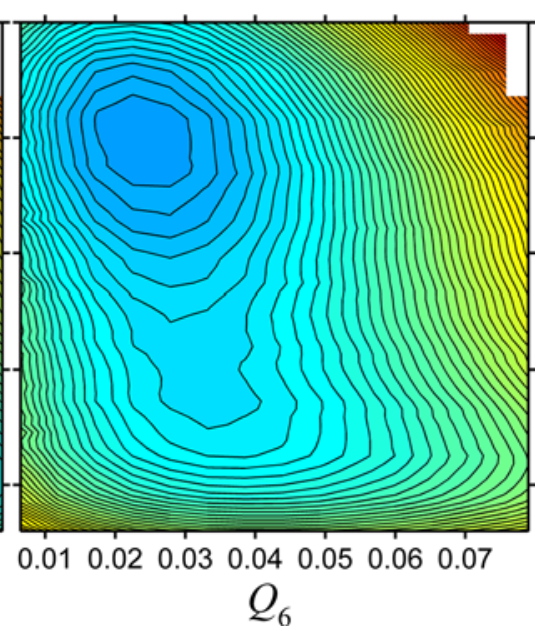

Figure 6. Free energy surfaces for the Jagla potential, at $T=0.0735 \varepsilon_{0} / k_{B}, N=300$ particles, and pressures of (a) $0.03 \varepsilon_{0} / r_{0}^{3}$, (b) $0.0322 \varepsilon_{0} / r_{0}^{3}$, and (c) $0.0343 \varepsilon_{0} / r_{0}^{3}$. The free energy surfaces in panels (a) and (c) were calculated by reweighting the surface shown in panel (b). Contour spacing is $1 k_{B} T$, and the statistical uncertainties are less than or approximately equal to $0.25 k_{B} T$ for most of the bins. Note that the free energy barrier separating the HDL and LDL basins is approximately $2 k_{B} T$, which is $1-2 k_{B} T$ lower than the barrier observed in ST2 water at the same- reduced temperature and $N=192$ molecules. 5,9

high-density high- $Q_{6}$ region. While this region of the free energy surface is not of great interest to the LLPT itself, studying this part of the surface may reveal alternate pathways to crystallization, or perhaps the existence of alternate crystal phases, and constitutes an area for future research.

The temperature of Figure 5 is well below the equilibrium freezing/melting line reported by $\mathrm{Xu}$ et al. ${ }^{43}$ indicating that the coexisting liquid phases are metastable with respect to the HCP crystal phase. Limmer and
Chandler have argued that the appearance of an LDL basin in free energy surface of ST2 is a non-equilibrium artifact associated with the disparity in relaxation times between density and $Q_{6}$, coupled with constraining the surface calculation to low (liquid-like) values of $Q_{6}{ }^{6}$ They argue that, upon allowing the free energy surfaces to 'truly' equilibrate, the LDL basin would disappear. Palmer et al., ${ }^{9}$ categorically disproved this assertion by sampling the free energy surface from the LDL to the stable crystal basin, and then reversibly sampling 
from the crystal basin back to the LDL by initiating the reverse simulations with a crystal configuration. Their reverse calculations incurred no change in the prediction of an LDL liquid state. We have performed a series of analogous calculations for the Jagla potential at the metastable condition $T=0.06 \varepsilon_{0} / k_{B}, P=0.02 \varepsilon_{0} / r_{0}^{3}$ in which we sample from the LDL to the stable HCP basin, and then sample in the reverse direction. While our first calculations did exhibit significant hysteresis in the vicinity of the crystal basin due to the formation of defects associated with the use of a cubic simulation box (the unit cell of HCP is not cubic, and hence the crystal phase is artificially constrained for this box geometry), there is no hysteresis for $Q_{6}<0.3$, with the LDL basin and saddle-point transition to the crystal phase perfectly maintained. We then implemented the same set of calculations with non-isotropic volume moves, ${ }^{65}$ allowing the box length to vary independently in all three dimensions, which resulted in much better agreement between the forward and reverse free energy surfaces. Refer to the "Appendix" for more details.

\subsection{Relaxation times}

A central theme in the arguments against the existence of an LLPT in ST2 water is the supposed vast separation of time scales between density and bond-orientational $\left(Q_{6}\right)$ relaxations. ${ }^{6}$ According to this hypothesis, the appearance of an LDL basin in the free energy surface of ST2 is caused by a non-equilibrium phenomenon associated with the coarsening of the incipient crystal phase, wherein density fluctuates widely in the early stages of coarsening prior to the development of long-range structural order. To support these arguments, Limmer and Chandler reported that the characteristic relaxation time (based on the autocorrelation function) of density $\left(\tau_{\rho}\right)$ is typically at least two orders of magnitude smaller ${ }^{6}$ than the characteristic relaxation time for $Q_{6}\left(\tau_{Q_{6}}\right)$ in the ST2 model of water. Limmer and Chandler regard this separation of time scales as 'self-evident'; ${ }^{16}$ however, the work of Palmer and coworkers ${ }^{9,66}$ directly contradicts this assertion. In the LDL phase of ST2 it has been shown ${ }^{9}$ that, across multiple sampling techniques, the autocorrelation of density and $Q_{6}$ decay in tandem, reaching zero at approximately the same time. The intermediate behavior of the autocorrelation functions varies slightly depending on the sampling method, with the density always decaying slightly faster than $Q_{6}$ for the state points studied; however, upon calculating the mean autocorrelation times via Equation (20), it is clear that density and bond-orientational order relax on the same time scale. In the HDL phase of ST2 the situation is actually quite different, ${ }^{66}$ with the interesting result that autocorrelations of $Q_{6}$ decay to zero significantly faster than those of density. This result is in direct conflict with the assertions of Limmer and Chandler (i.e., $\tau_{Q_{6}}>>\tau_{\rho}$ ), and is correspondingly at odds with previous autocorrelation curves published by those authors. ${ }^{16}$ At this time, the reason for this discrepancy remains unclear.

We will now examine the aforementioned relaxation times, $\tau_{\rho}$ and $\tau_{Q_{6}}$ associated with the HDL and LDL phases of the Jagla potential in order to facilitate comparisons with ST2 water. It has already been documented that, at temperatures well below the critical temperature, the HDL phase of the Jagla model is significantly (i.e., up to 1-2 orders of magnitude) less diffusive than the LDL phase. ${ }^{43}$ This result is in agreement with our own observations that simulations of the HDL phase exhibit longer-wavelength fluctuations in thermodynamic quantities, and tend to take significantly longer to equilibrate than LDL simulations. This is the opposite of ST2, for which the LDL is the more slowly relaxing phase. In Figure 7, we plot the autocorrelation functions of density and $Q_{6}\left(C_{\rho}(t)\right.$ and $C_{Q_{6}}(t)$, respectively) at the coexistence pressure for $T=0.06 \varepsilon_{0} / k_{B}$.

It is immediately apparent that the autocorrelation functions in the HDL phase are drastically different, while in the LDL phase they are almost identical at these particular conditions. Moreover, in the HDL phase it is the autocorrelation in density which relaxes significantly more slowly than that of $Q_{6}$. Hence, this serves as another clear example of a case where the conjecture $\tau_{Q_{6}}>\tau_{\rho}$ is invalid. In this particular case, we have the completely opposite scenario $\left(\tau_{\rho}>>\tau_{Q_{6}}\right)$, in qualitative agreement with results recently obtained by Palmer ${ }^{66}$ for the HDL phase of ST2.

In order to quantify the autocorrelation times, we apply Equation (20) and determine that the calculated autocorrelation times for the LDL phase are $\tau_{\rho}=$ $970 \mathrm{MC}$ sweeps and $\tau_{Q_{6}}=800 \mathrm{MC}$ sweeps, while the autocorrelation times for the HDL phase are $\tau_{\rho}=$ 25, $000 \mathrm{MC}$ sweeps and $\tau_{Q_{6}}=1,700 \mathrm{MC}$ sweeps. In addition to coexistence conditions, we were also interested in investigating how the relaxation times of each liquid vary with pressure along an isotherm in both the stable and metastable regimes. We therefore compute values of $\tau_{\rho}$ and $\tau_{Q_{6}}$ in each phase at $T=0.06 \varepsilon_{0} / k_{B}$ with pressures traversing the stable and metastable regions of each liquid, as shown in Figure 8. Note that we are using the terms 'stable' and 'metastable' here to refer to the relative stabilities of the HDL and LDL phases, and not to the overall thermodynamic stability of the phase (at $T=0.06 \varepsilon_{0} / k_{B}$ both liquid phases are metastable with respect to the HCP crystal phase for the range of pressures studied). 
HDL

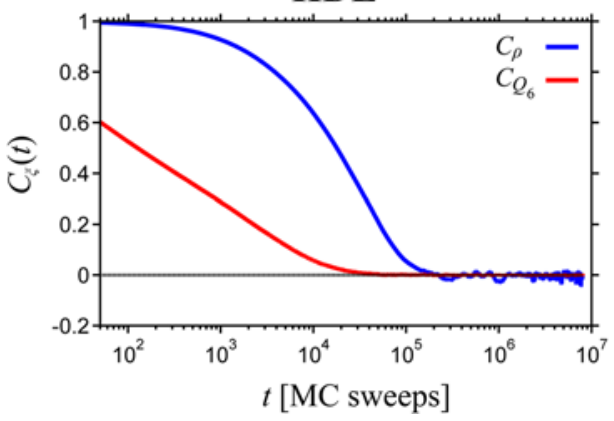

LDL

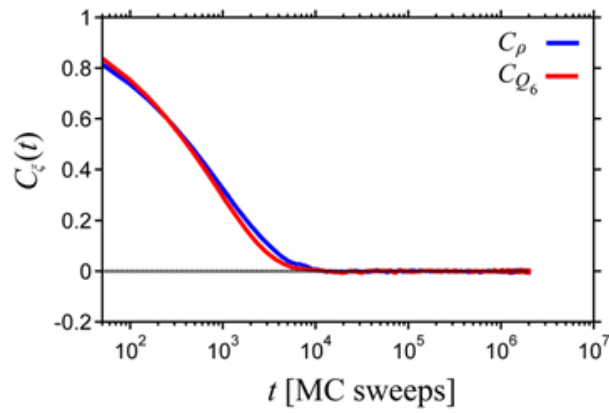

Figure 7. Autocorrelation functions of density and $Q_{6}$ for unconstrained NPT MC simulations at coexistence conditions $T=0.06 \varepsilon_{0} / k_{B}$, and $P=0.02 \varepsilon_{0} / r_{0}^{3}(N=300$ particles $)$. The curves shown are the average of 20 independent simulations. Notice the large disparity between the autocorrelation functions in the HDL, while in the LDL they are almost identical at these conditions.

As observed in Figure 8, the behaviors of $\tau_{\rho}$ and $\tau_{Q_{6}}$ are drastically different for the HDL and LDL phases. In the HDL the relaxation times of density are an order of magnitude larger than those of $Q_{6}\left(\tau_{\rho}>>\tau_{Q_{6}}\right)$ over the entire range of pressures studied. Over this range of pressures, spanning from the stable HDL region well into the metastable HDL region, the loci of $\tau_{\rho}$ and $\tau_{Q_{6}}$ do not vary drastically with pressure, and both generally follow the same trend. In the LDL the relaxation times of density and $Q_{6}$ are comparable, and interestingly we observe an inversion in which variable relaxes more slowly. Because these simulations are carried out in the NPT ensemble, as we explored pressures closer and closer to the spinodal pressures of each phase there eventually came a point beyond which the phase of interest could not be sampled for a sufficient length of time before it spontaneously changed to the stable liquid phase (in analogy to the hysteresis observed in Figure 2), hence we were unable to perform measurements arbitrarily close to the spinodals. We also performed a similar analysis at $T=0.065 \varepsilon_{0} / k_{B}$ and observed qualitatively identical behavior in $\tau_{\rho}$ and $\tau_{Q_{6}}$ for both phases (see "Appendix").

Figure 8 provides a comprehensive illustration of how relaxation times associated with an LLPT vary as a function of pressure in the Jagla model. A similar analysis with the ST2 model of water could prove instructive, and would certainly help to better understand the relative relaxation rates of density and bond-orientational order in that model, particularly within the context of an LLPT.
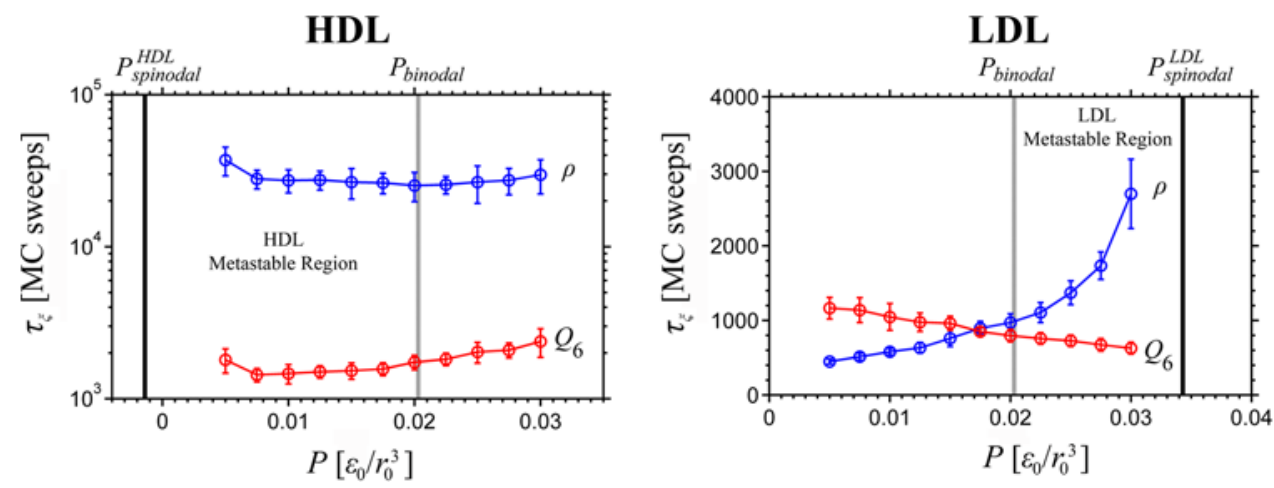

Figure 8. Characteristic relaxation times of density and $Q_{6}$ as a function of pressure for unconstrained NPT MC simulations at $T=0.06 \varepsilon_{0} / k_{B}(N=300$ particles $)$. The pressure $P_{\text {binodal }}$ (grey hashed line) is the calculated liquid-liquid coexistence pressure at this temperature, and $P_{\text {spinodal }}$ (solid black line) denotes the spinodal pressure of the phase (HDL, LDL). For pressures between $P_{\text {binodal }}$ and $P_{\text {spinodal }}$ (denoted "Metastable Region"), the liquid phase of interest is metastable with respect to the other liquid phase. The data points are the average of the relaxation times, computed individually for each of the 20 independent simulations via Equation (20), and the lines are guides to the eye. Error bars represent the standard deviation of the relaxation times for the 20 independent runs. 


\subsection{Liquid-liquid surface tension}

Of inherent importance to the study of phase transitions is the interfacial tension, $\gamma$. Recently, Palmer et al.,${ }^{9}$ produced a preliminary estimate of the liquid-liquid surface tension in the ST2 model at $T_{r}=0.96$ of approximately $2 \mathrm{~mJ} / \mathrm{m}^{2}$. Liquid-liquid surface tension calculations for the Jagla potential have been provided in the literature ${ }^{54}$ for a range of reduced temperatures $T_{r} \approx 0.8-0.96$, but these calculations were performed by evaluating elements of the pressure tensor obtained during molecular dynamics simulations in which an explicit HDL-LDL interface is formed. This method is best suited for low-to-moderate reduced temperatures because as one approaches the critical temperature fluctuations grow and it becomes difficult to maintain a stable interface. ${ }^{67}$

We evaluate the liquid-liquid surface tension of the Jagla potential at 3 reduced temperatures near the critical (a)

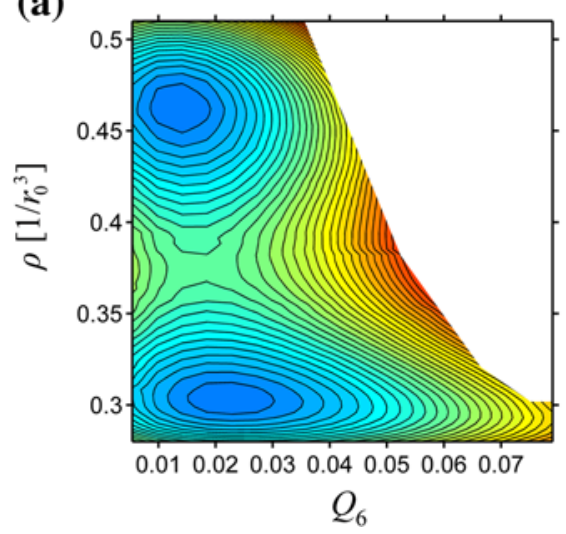

(b)

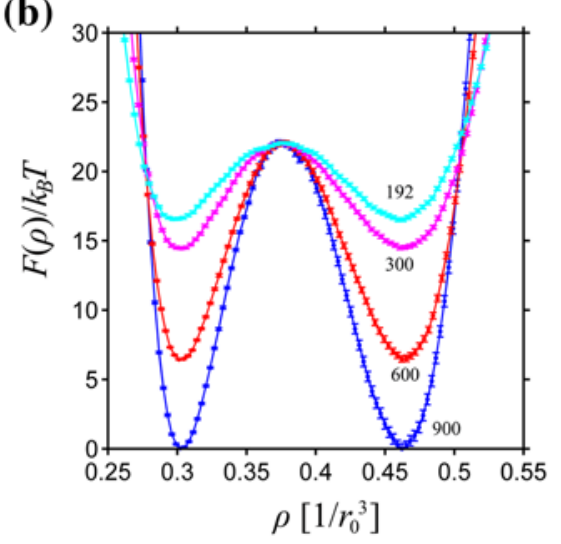

(c)

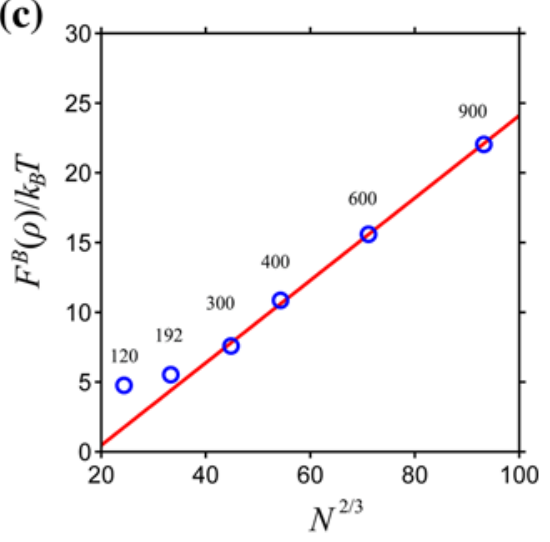

Figure 9. Calculation of HDL-LDL free energy barrier height at coexistence, $T=0.07 \varepsilon_{0} / k_{B}, P=0.0292 \varepsilon_{0} / r_{0}^{3}$. (a) Free energy surface for $N=900$, contours $=2 k_{B} T$. (b) Contracted free energy surfaces for select system sizes $N=192,300,600$, and 900. Error bars in (b) are the standard deviation of 200 contracted bootstrapped free energy surfaces. ${ }^{62}$ (c) System-size scaling of the free energy barrier height, $F^{B}$, for all system sizes studied. The system-size dependence of the free energy barrier height begins to follow the expected $N^{2 / 3}$ scaling for $N \geq 300$ particles. The red line in (c) is a linear fit to the data points $N \geq 300$. Where necessary, free energy surfaces were reweighted slightly in pressure via Equation (17) to achieve coexistence between the HDL and LDL phases.
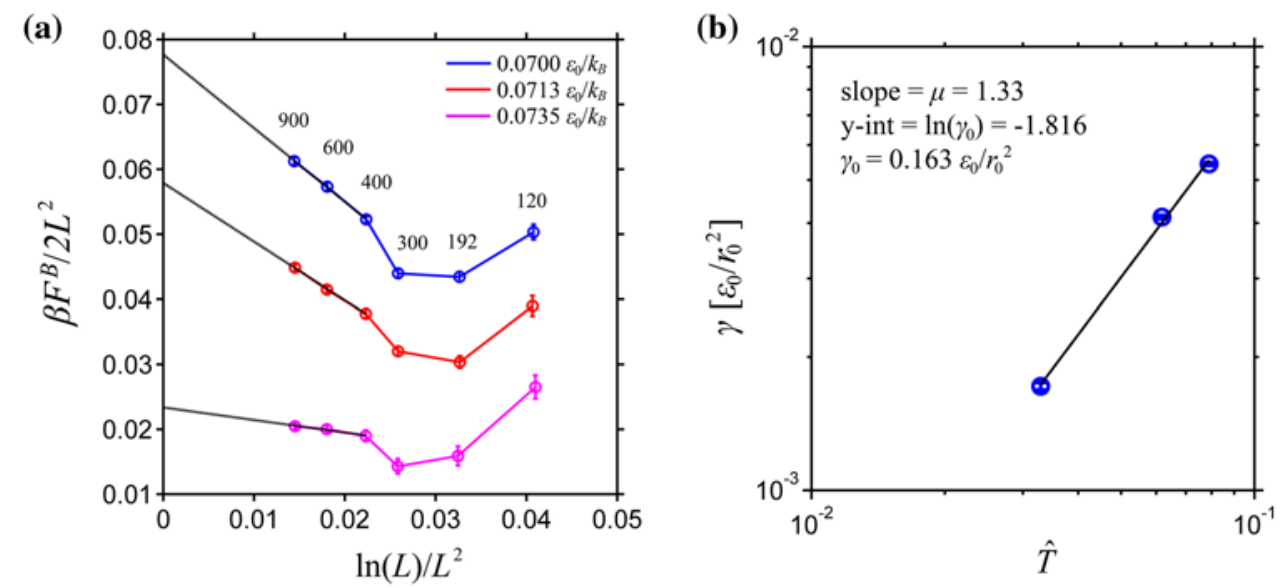

Figure 10. Calculation of the liquid-liquid surface tension, $\gamma$, of the Jagla potential. (a) System-size dependence of the effective surface tension for three different temperatures. For all three temperatures, the three largest system sizes $(N=400,600,900)$ show linear behavior, and hence these system sizes were utilized for the linear fit at each temperature which are represented as blacklines. The y-intercept of the linear fit is the extrapolation of the effective surface tension to infinite system-size for each temperature, $\gamma / k_{B} T$. Note that the ordinate in (a) has units of $r_{0}^{-2}$. (b) Surface tension as a function of scaled temperature, $\hat{T}=\left|\left(T-T_{C}^{L L}\right) / T_{C}^{L L}\right|=\left|T_{r}-1\right|$. A linear fit to the logarithm of Equation (22), illustrated by the black line, was performed in order to obtain the critical exponent, $\mu$. The calculated value of $\mu$ is 1.33 , which is only a $6 \%$ discrepancy with the expected value 1.26 . 
point $\left(T_{r}=0.92,0.94,0.97\right)$, where the explicitinterface method ${ }^{54}$ becomes problematic, by evaluating the system-size dependence of the free energy barrier separating the coexisting (HDL and LDL) basins, as described in the literature. ${ }^{67-69}$ In order to implement this method, we first calculate free energy surfaces for multiple system sizes $(N=120,192,300,400,600,900)$ at the coexistence pressure for the temperature of interest. An example free energy surface is shown in Figure 9(a) for $N=900, T_{r}=0.92$. The free energy surfaces were then contracted along the $Q_{6}$ dimension via Equation (18) in order to obtain $F(\rho)$, and the height of the barrier separating the HDL and LDL basins, $F^{B}$, was measured along with the density at which the maximum occurs, $\rho^{B}$. As shown in Figure 9(c), for sufficiently large systems the LLPT obeys the $N^{2 / 3}$ scaling characteristic of first-order phase transitions.

According to the finite-size scaling formalism of Binder $^{67-69}$ the effective surface tension for a finite-size system, $\gamma_{L}$, can be written as:

$$
\beta \gamma_{L}=\frac{\beta F_{L}^{B}}{2 L^{2}}=c_{1} \frac{1}{L^{2}}+c_{2} \frac{\ln L}{L^{2}}+\beta \gamma
$$

where, $L$ is the linear extent of the system which we define as $L=\left(N / \rho^{B}\right)^{1 / 3}, F_{L}^{B}$ is the free energy barrier height for the system of linear extent $L, \gamma$ is the infinite system-size (thermodynamic limit) surface tension, $c_{1}$ and $c_{2}$ are constants, and $\beta=1 / k_{B} T$. Note that the factor of 2 in the denominator of $\beta F_{L}^{B} / 2 L^{2}$ arises from the formation of two interfaces in a simulation with periodic boundary conditions.

It is clear from Equation (21) that the term $\beta F_{L}^{B} / 2 L^{2}$ becomes linear in the scaling variable $\ln (L) / L^{2}$ as the system size (and hence, $L$ ) approaches infinity. Therefore, the procedure for determining the surface tension in the thermodynamic limit is to plot $\beta F_{L}^{B} / 2 L^{2}$ vs. $\ln (L) / L^{2}$ for progressively larger system sizes, and when the system sizes are large enough such that the data transitions to a linear regime, extrapolate the linear data to $\ln (L) / L^{2}=0$. The y-intercept of this linear extrapolation is $\beta \gamma$, the infinite system-size surface tension scaled by $k_{B} T$. This procedure is illustrated in Figure 10(a). Note that we observe the effective interfacial tension is a non-monotonic function of system size, as was found to occur for the three dimensional Ising model $^{70}$ and the liquid-vapor transition of the Lennard-Jones potential. ${ }^{69}$ It is believed that this nonmonotonicity occurs due at least in part to the interaction between two interfaces in the periodic simulation box for smaller systems. ${ }^{69}$

In the vicinity of a critical point, the surface tension displays the following universal behavior: ${ }^{69}$

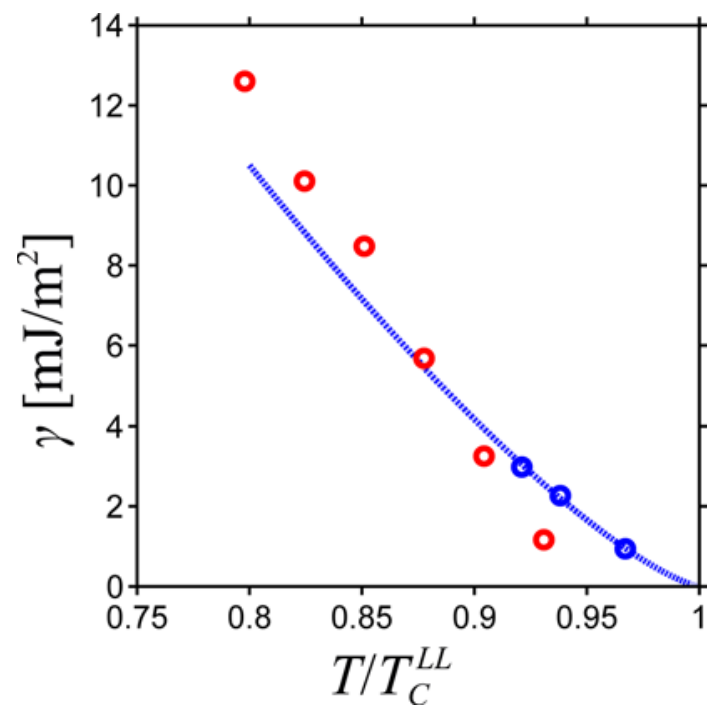

Figure 11. Liquid-liquid surface tension vs. reduced temperature. The surface tensions calculated in this work via free energy barrier finite-size scaling are represented by the blue circles, while the surface tensions calculated by Buldyrev et al., ${ }^{54}$ via the explicit-interface method are reproduced (with permission) as red circles. Error bars for our data are smaller than the symbol size. All surface tensions were converted into physical units with the conversion factors of $\mathrm{Xu}$ et al. ${ }^{47}$ The dotted blue curve is the asymptotic scaling predicted by Equation (22), employing our calculated values of $\mu=1.33$, and $\gamma_{0}=89.4 \mathrm{~mJ} / \mathrm{m}^{2}$. Substituting the expected value of $\mu=1.26$ imparts slightly higher curvature, which slightly increases the predicted asymptotic-locus of surface tensions. We note that the calculations of Buldyrev et al., 54 were performed with a slightly modified energy parameterization of $U_{R} / U_{0}=3.56$ (see Model and Numerical Methods section for more details), hence for consistency we utilized the reported 41 $T_{C}^{L L}$ of the original Jagla parameterization to calculate the reduced temperatures for our data, and the reported ${ }^{53} T_{C}^{L L}$ of the modified parameterization to calculate the reduced temperatures of Buldyrev et al. ${ }^{54}$ This slight difference in energy parameterization may also impart slight differences to the calculated surface tensions at corresponding reduced temperatures; however, the asymptotic critical scaling should still be observed.

$$
\gamma= \begin{cases}\gamma_{0} \hat{T}^{\mu} ; & T<T_{C} \\ 0 ; & T>T_{C}\end{cases}
$$

where, $\hat{T}=\left|\left(T-T_{C}\right) / T_{C}\right|, \mu$ is the universal critical exponent $^{71}$ for surface tension, which is equal to 1.26 for the 3-dimensional Ising universality class, ${ }^{68} \gamma_{0}$ is the system-dependent coefficient of proportionality, and $T_{C}$ denotes the critical temperature, which in the context of the liquid-liquid critical point is $T_{C}^{L L}$. We also note that the finite-size scaling analysis of Gallo and Sciortino ${ }^{72}$ 
has verified that the Jagla potential indeed belongs to the Ising universality class. As shown in Figure 10(b), the calculated value of $\mu$ is 1.33 , which is in good agreement (6\% discrepancy) with the expected value of 1.26.

The surface tensions reported in Figure 10(b) are in Jagla units. In order to facilitate a comparison to water and other real substances, we employ the conversion factors of $\mathrm{Xu}$ et al. ${ }^{47}$ to obtain the surface tension in units of $\mathrm{mJ} / \mathrm{m}^{2}$ as shown in Figure 11 . We see that our calculated surface tensions range from approximately $1-3 \mathrm{~mJ} / \mathrm{m}^{2}$ for a corresponding reduced temperature range of $0.97-0.92$, which is quite similar to the reported value of $2 \mathrm{~mJ} / \mathrm{m}^{2}$ for the $\mathrm{ST} 2$ model at a reduced temperature of 0.96 .

We also compare our results with previously reported liquid-liquid surface tensions for the Jagla potential. ${ }^{54}$ Referring to Figure 11, we see that the calculations of Buldyrev et al. ${ }^{54}$ which require the maintenance of an explicit interface, do not follow the asymptotic scaling predicted by Equation (22) in the vicinity of the critical point, and the highest-temperature data points under-predict the surface tension as the critical point is approached. As expected, the surface tensions calculated via finite-size scaling of the free energy barrier appear closer to the theoretically prescribed behavior, with the surface tension vanishing as described by Equation (22). While we plot our fit of Equation (22) over the reduced temperature range $0.8-1$ for illustrative purposes, we note that this scaling relation is only expected to hold in the general vicinity of the critical point, and hence we expect the explicit-interface calculations ${ }^{54}$ are accurate at conditions remote from the critical point.

\section{Conclusions}

In this work, we provide rigorous free energy surface calculations which demonstrate the existence of a liquidliquid phase transition in the Jagla potential. While equation of state and finite-size scaling calculations have been performed to date, free energy surface calculations (which represent the free energy of a system as a function of density and an additional order parameter that distinguishes liquid and crystal configurations) have become the gold standard in the LLPT literature. ${ }^{4-9}$ We find that free energy surfaces of the Jagla model are qualitatively identical to those calculated ${ }^{5-9}$ for the ST2 model of water, even including the noticeably larger width in the $Q_{6}$ dimension of the LDL basin as compared to the HDL basin. Because the LLCP is located slightly above the freezing line, ${ }^{43}$ a significant portion of the LLPT locus is only moderately metastable with respect to crystallization. This fact, coupled with the relatively fast relaxation dynamics in the vicinity of the LLPT and the computationally inexpensive nature of the Jagla potential, allowed us to explore free energy surfaces at reduced temperatures well below those accessible for the ST2 model of water. Our calculated free energy surfaces show excellent agreement with our own equation of state calculations, which lends further confidence to the several equation of state studies of the Jagla potential in the literature. ${ }^{40-43}$ We note that, at temperatures well below the LLCP, as in Figure $5\left(T_{r}=0.79\right)$, we found it difficult to sample the region of high density and high $Q_{6}$. Although this region of the free energy surface is not of particular importance to the LLPT itself, a more focused effort to resolve this portion of the free energy surface may reveal alternative pathways to crystallization (particularly at higher pressures, beyond the LDL spinodal, where crystallization information is lacking in this model ${ }^{43,47}$ ), and as such it constitutes an area for future research.

In addition to our free energy analysis, we also performed a brief analysis of bond-orientational and translational order in the context of the LLPT. We point out that, while the HDL phase is the lower-entropy phase in the Jagla model, it in fact has lower bond-orientational order than the LDL phase in these finite-size simulations. However, we find that the HDL phase has a higher degree of translational order than the LDL phase, which appears to be clearly reflected in the entropy, truncated at the two-body interactions.

We performed extensive calculations of the characteristic relaxation times of density and $Q_{6}$ for the HDL and LDL liquids in order to compare the relaxation time scales over a range of conditions where each liquid was stable, at coexistence, and metastable with respect to the other liquid. These relaxation time calculations are of particular relevance because they have been a centerpiece of arguments, ${ }^{6,16}$ which argue against the possible existence of pure-component metastable liquid-liquid phase separations. Hence, we performed our calculations for the Jagla potential at temperatures for which the LLPT is metastable with respect to crystallization, and make appropriate comparisons with ST2. Contrary to the assertions of Limmer and Chandler, ${ }^{6,16}$ and in agreement with the findings of Palmer et al., 9,66 for ST2, we do not find any range of conditions for which the relaxation of $Q_{6}$ is orders of magnitude slower than that of density in the Jagla model. Interestingly, we find exactly the opposite scenario for the HDL phase (namely $\tau_{\rho}>\tau_{Q_{6}}$ ), which is also in agreement with observations by Palmer for the HDL phase of ST2. ${ }^{66}$ For the LDL phase of Jagla, the relaxation times are much more comparable, and as we vary pressure along an isotherm we observe an inversion in which the variable relaxes 
more slowly. When comparing the two phases with one another, it is clear that the HDL phase is much more slowly relaxing owing to the very slow relaxations in density. This result is in contrast with the ST2 model for which the LDL phase is more slowly relaxing. The aforementioned results for relaxation rates are shown to apply across multiple temperatures (see "Appendix" for results at higher temperature), and categorically refute the notion that a separation of time scales of the sort $\tau_{Q_{6}}>>\tau_{\rho}$ is "self-evident" at supercooled conditions. While relaxation times of density and $Q_{6}$ have been calculated for the ST2 potential in many different studies, there has yet to be a comprehensive investigation of how these relaxation times change with varying temperature and pressure. Such a calculation may prove instructive in better understanding the relative relaxation rates of these quantities within the broader context of the LLPT.

The surface tension is of intrinsic importance to the study of phase transitions, yet the calculation of surface tensions associated with a pure-component liquid-liquid phase transition is still relatively unexplored. One estimation has been made for the LLPT of ST $2,{ }^{9}$ and several state points were calculated ${ }^{54}$ for the LLPT of Jagla by simulating an explicit interface in a very large system and evaluating elements of the pressure tensor. Since the explicit-interface method becomes problematic in the vicinity of a critical point, we performed a free energy barrier scaling analysis ${ }^{67-69}$ at three reduced temperatures near the critical point in order to calculate the infinite system-size surface tension. Our calculated surface tensions very closely follow the predicted asymptotic critical scaling, ${ }^{69}$ and comparison with the previously reported explicit-interface results ${ }^{54}$ show considerable improvement in the vicinity of the critical point, as expected. Our free energy surface calculations show that obtaining results at reduced temperatures as low as 0.79 is computationally tractable for the Jagla potential (Figure 5). Hence, one can in principle use free energy barrier scaling to determine liquid-liquid surface tensions for the Jagla potential at conditions more remote from the critical point than those explored here. We note that a study dedicated to calculating liquid-liquid surface tensions in the Jagla potential may be better suited to an approach which directly calculates a free energy profile as a function of density alone $^{67,69}$ (i.e., $F(\rho)$ ), provided suitable precautions are taken to monitor $Q_{6}$ and ensure that crystallization does not occur.

Despite their very different functional forms, it has been long appreciated ${ }^{43,46}$ that the isotropic Jagla potential qualitatively reproduces several of the anomalous behaviors found in considerably more complex models of network-forming fluids such as water. Throughout this work, we have made it a point to compare our results for the Jagla model with those of the ST2 model of water in order to emphasize key similarities and differences between the two, particularly within the context of the LLPT. It is our hope that this study will contribute towards an elucidation of which aspects of a model are necessary, sufficient, and/or prohibited for the realization of pure-component liquid-liquid phase separation.

\section{Acknowledgements}

It is a privilege to dedicate this work to the memory of Professor Charusita Chakravarty, in admiration of her outstanding contributions to science, and in gratitude for her friendship. She was an extraordinary human being and will remain an inspiration throughout the broad international community of liquid-state and condensed matter scientists. We would like to thank Jeremy Palmer for several invaluable discussions during the development of this work, and Sergey Buldyrev for providing us with the liquid-liquid surface tension data. PGD gratefully acknowledges the financial support of the US National Science Foundation (grant CHE-1213343).

\section{Appendix}

\section{A.1 Free energy sampling to the crystal phase}

In a recent publication, Palmer et al. ${ }^{9}$ sampled the free energy surface of ST2 to the stable crystal basin and reversibly sampled back to the LDL phase. ${ }^{9}$ These calculations categorically refuted the assertion ${ }^{6}$ that the appearance of an LDL basin in preliminary free energy surface calculations ${ }^{5}$ of ST2 water was due to the disparity in relaxation times between density and $Q_{6}$, coupled with constraining free energy surface calculations to a liquid-like range of $Q_{6}$. We performed analogous calculations for the Jagla potential at the condition $T=$ $0.06 \varepsilon_{0} / k_{B}, P=0.02 \varepsilon_{0} / r_{0}^{3}$, which is well below the reported equilibrium freezing line. ${ }^{43}$ As shown in the Figure 12, the crystal basin is of significantly lower free energy than the HDL and LDL basins as one would expect at substantially supercooled conditions. However, we note that the crystalline configurations sampled the high- $Q_{6}$ region were defect-rich due to the use of a cubic simulation box (the thermodynamically favored HCP crystal phase does not have a cubic unit cell), and hence the depth of the free energy basin is underpredicted. ${ }^{73}$ We will address this point below, but we note here that this is not of significant consequence, as our primary concern was with whether the LDL basin and LDL-crystal transition barrier would be identically recovered upon performing calculations which were initiated with a crystal configuration, as explained below. 


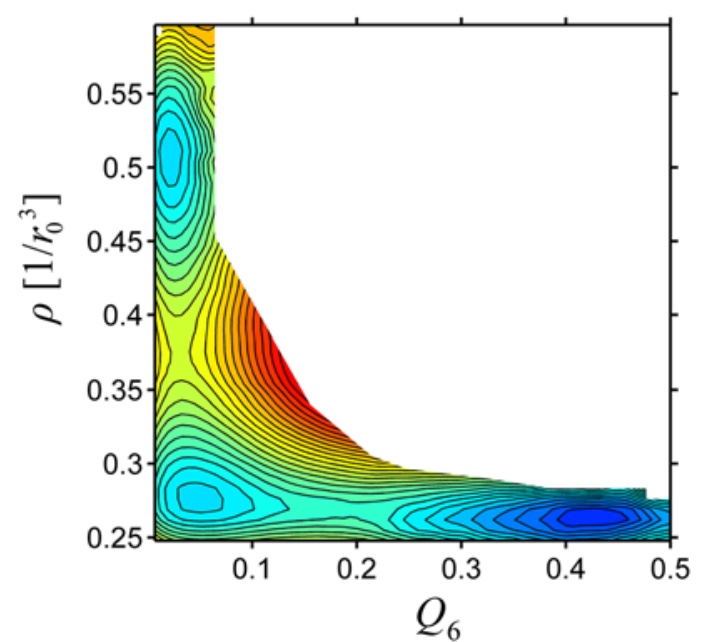

Figure 12. Free energy surface for the Jagla potential, at $T=0.06 \varepsilon_{0} / k_{B}, P=0.02 \varepsilon_{0} / r_{0}^{3}$, $N=300$ particles. All simulations were carried out with a standard cubic system geometry and periodic boundary conditions. Contour spacing is $5 k_{B} T$, and the statistical uncertainties are less than, or on the order of $1-2 k_{B} T$ for most of the bins, with bins near the crystal basin exhibiting uncertainties as large as $5 k_{B} T$. The LLPT is clearly metastable with respect to crystallization at this condition; however, defects in the HCP crystal associated with the artificial constraint of a cubic simulation box geometry produce a crystal basin which is too shallow.

Once the 'forward' (LDL $\rightarrow$ crystal) free energy surface shown in Figure 12 was calculated, we computed the free energy surface in the 'reverse' (crystal $\rightarrow$ LDL) direction by initiating the reverse simulations with a crystal configuration taken from a simulation near the minimum of the crystal basin in Figure 12. Free energy profiles obtained by contracting the forward and reverse free energy surfaces are shown in Figure 13(a). As expected, there is significant hysteresis in the vicinity of the crystal basin associated with the use of a cubic simulation box and the presence of ample defects in the starting crystal configuration. However, the purpose of this preliminary calculation was not to accurately determine the precise free energy change upon transitioning from the LDL phase to the crystal phase, but rather to observe whether the LDL basin would be recovered upon performing the calculations which were initiated with a crystal configuration. Indeed, for $Q_{6}<0.3$ there is no hysteresis within statistical uncertainties and the LDL basin is recovered identically.

In order to obtain better agreement between the crystal basin depths of the forward and reverse calculations, we implemented non-isotropic volume moves, ${ }^{65}$ allowing the box length to vary independently in all three dimensions while maintaining right angles between all vertices. Because the application of non-isotropic volume moves in liquid (i.e., low $Q_{6}$ ) simulations could lead to unfettered box elongation, which would cause erroneous results if one of the box dimensions became shorter than the cutoff distance of the model, we implemented cubic simulation box geometry for $Q_{6} \leq 0.2$. As shown in Figure 13(b), the agreement between the forward and reverse calculations is much better, with the depths of the crystal basin minima being statistically indistinguishable (within one standard deviation). However, we note that for the reverse case the $Q_{6}$ value of the crystal basin minimum is shifted lower by approximately 0.02 , and in both cases the crystal basin minimum occurs at a $Q_{6}$ value lower than the expected value for a perfect HCP lattice ${ }^{57}(0.485)$, which indicates that there are still measurable defects. Hence, while including non-isotropic volume moves significantly improves the agreement between the forward and reverse simulations, longer equilibration times and/or more sophisticated sampling methods ${ }^{74}$ are needed in order to see an improvement in reversibility for $Q_{6}>0.3$. We reiterate however that the purpose of these calculations is to demonstrate that, deep within the supercooled region, the Jagla potential's LDL phase is not a non-equilibrium artifact associated with the formation of the stable crystal phase.

\section{A.2 Relaxation times at alternative temperatures}

In this work, we study how the characteristic relaxation times of density and bond-orientational order vary with pressure along an isotherm. In Figure 8, we illustrated the behavior at $T=0.06 \varepsilon_{0} / k_{B}$ with pressures traversing the stable and metastable regions of each liquid (we point out once again that the terms 'stable' and 'metastable' here to refer to the relative stabilities of the HDL and LDL phases, and not to the overall thermodynamic stability of the phase). We also performed similar calculations a higher reduced temperature, $T=$ $0.065 \varepsilon_{0} / k_{B}\left(T_{r}=T / T_{C}^{L L}=0.86\right)$, in order to check whether the interesting observations at $T=0.06 \varepsilon_{0} / k_{B}$ are reproduced. Referring to Figure 14, we note that the same qualitative behavior is indeed observed for the relaxation times of density and $Q_{6}$. In the HDL the relaxation times of density are approximately two orders of magnitude larger than those of $Q_{6}\left(\tau_{\rho}>>\tau_{Q_{6}}\right)$ over the entire range of pressures studied, and furthermore the loci of $\tau_{\rho}$ and $\tau_{Q_{6}}$ do not vary drastically with pressure, with both generally following the same trend. In the LDL the relaxation times of density and $Q_{6}$ are much more similar over the majority of the pressures studied, with the exception of the vicinity of the spinodal. As 

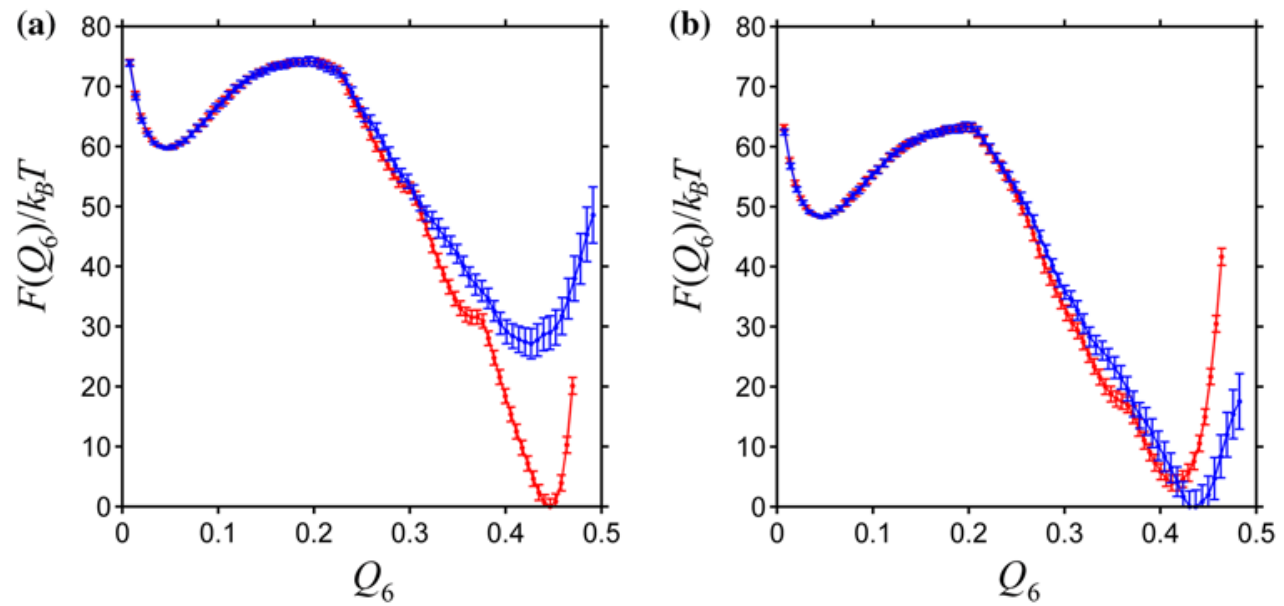

Figure 13. Contracted free energy for the Jagla potential, at $T=0.06 \varepsilon_{0} / k_{B}$, $P=0.02 \varepsilon_{0} / r_{0}^{3}, N=300$ particles. Simulations in the "forward" direction (blue) were initiated from liquid configurations, while simulations in the 'reverse' direction (red) were initiated with a single crystal configuration taken from near the minimum of the crystal basin of the corresponding 'forward' calculation. The free energy contractions were performed for densities $\leq 0.325 r_{0}^{-3}$ so as to exclude the HDL basin.The free energy profiles are shifted such that they have the same free energy at the minimum of the LDL basin. (a) Standard cubic system geometry, as shown in Figure A.1.1. (b) Rectangular prism box geometry, where the length of all three box sides are allowed to vary independently of one another for simulations with $Q_{6}>0.2$. For $Q_{6} \leq 0.2$, cubic box geometry was employed. Notice that in both cases, (a) and (b), there is no hysteresis (within statistical uncertainties) for $Q_{6}<0.3$. As expected, significant improvement in the high- $Q_{6}$ agreement between the forward and reverse calculations is found with the incorporation of non-isotropic volume moves, although more sophisticated sampling techniques and longer simulation times may be required in order to obtain perfect agreement in this region. Error bars are the standard deviation of 200 contracted bootstrapped free energy surfaces. ${ }^{62}$
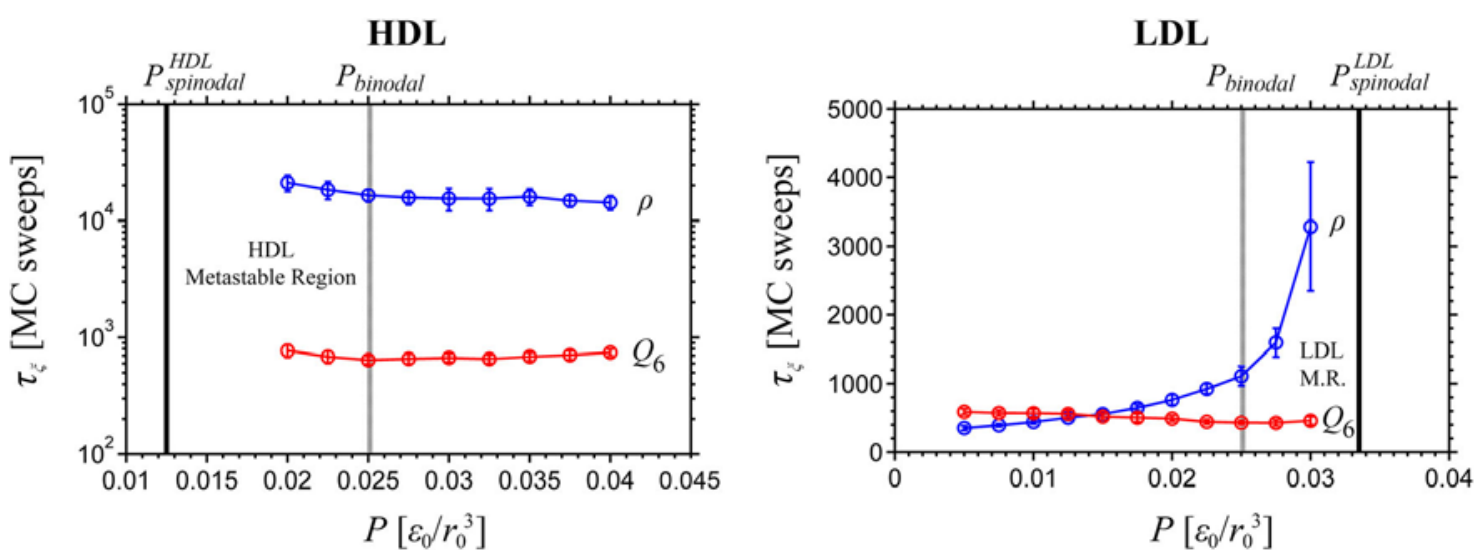

Figure 14. Characteristic relaxation times of density and $Q_{6}$ as a function of pressure for unconstrained NPT MC simulations at $T=0.065 \varepsilon_{0} / k_{B}\left(N=300\right.$ particles). The pressure $P_{\text {binodal }}$ (grey hashed line) is the calculated liquid-liquid coexistence pressure at this temperature, and $P_{\text {spinodal }}$ (solid black line) denotes the spinodal pressure of the phase (HDL, LDL). For pressures in between $P_{\text {binodal }}$ and $P_{\text {spinodal }}$ (denoted "Metastable Region" or abbreviated as "M.R."), the liquid phase of interest is metastable with respect to the other liquid phase. The data points are the average of the relaxation times computed individually for each of the 20 independent simulations via Equation (20), and the lines are a guide to the eye. Error bars represent the standard deviation of the relaxation times for the 20 runs.

at $T=0.06 \varepsilon_{0} / k_{B}$, we observe an inversion in which variable relaxes more slowly for the LDL. Note that, because we are operating at a higher reduced tempera- ture (i.e., closer to the critical temperature), the HDL and LDL metastable regions are narrower, and the approach to the spinodals is more challenging. 


\section{A.3 Free energy surfaces for the generalized family of Jagla potentials}

The Jagla potential is a fascinatingly simple model which captures various anomalies exhibited by models of much greater complexity, such as those of atomistic water. While the original parameterization of the Jagla potential captures many of the key behaviors of networkforming liquids such as water, one may adjust the potential's parameters (by varying the length scales $r_{1}$ and $r_{2}$, and the depth of the potential-minimum located at $r_{1}$ ) so as to continuously transform it to a ramp-based approximation of the Lennard-Jones potential. This is interesting because, unlike water, the liquid phase of the Lennard-Jones model is an archetypical 'simple liquid'. Whence, by exploring the generalized family of Jagla potentials one may study the change in phenomenology as the model varies from the original (anomalous) Jagla model to the (simple) Lennard-Jones model. This continuous transformation should hypothetically incur a loss of all of the anomalous properties of the ramppotential, as well as the disappearance of the LLPT, which does not exist for the Lennard-Jones model. This type of calculation was first performed by Gibson and Wilding, ${ }^{42}$ who utilized equation of state calculations in the NPT ensemble to examine the phase behavior of these models, along with multicanonical Monte Carlo, and a 'crude' version of finite-size scaling analysis to locate the LLPT and estimate critical parameters. More recently, Luo et al., ${ }^{50}$ performed an analogous study on the Jagla potential via discrete molecular dynamics in which they instead examine the behavior of the Widom line at conditions above the critical temperature for multiple potential parameterizations. Although each study differed slightly by the manner in which the Jagla potential was modified, both efforts yielded similar results: upon decreasing the ratio $r_{1} / r_{0}$, the LLCP moves to lower temperatures and higher pressures, and the slope of the liquid-liquid coexistence line decreases, eventually becoming negative. Furthermore, Gibson and Wilding ${ }^{42}$ explicitly report a concomitant increase in the temperature of spontaneous crystallization (i.e., the loss of liquid stability) upon decreasing $r_{1} / r_{0}$. We note once again that these authors refer to the onset of spontaneous crystallization as the 'freezing point', which is not to be confused with the equilibrium freezing/melting point. Both studies ${ }^{42,50}$ also independently report that at a length scale ratio $r_{1} / r_{0} \approx 1.59$ the LLCP becomes prohibitively difficult to locate due to the onset of spontaneous crystallization near the critical point. This length scale ratio also happens to be in the immediate vicinity of where the slope of the coexistence line changes from positive to negative.
We will now study the generalized family of Jagla potentials described by Gibson and Wilding ${ }^{42}$ via free energy surface calculation. By evaluating the free energy surfaces, we can unambiguously elucidate the existence of an LLPT while avoiding the crystallization issues which plagued previous studies in the vicinity of $r_{1} / r_{0} \approx$ 1.59. Note that for each value of $r_{1} / r_{0}$ studied, we choose all other model parameters to be the same as those used by Gibson and Wilding ${ }^{42}$ (refer to Table 1 of that work for additional model parameters).

The state points for calculation of free energy surfaces were chosen as follows. For a given value of $r_{1} / r_{0}$, the critical temperature and pressure were extracted from Figure 4 of Gibson and Wilding, ${ }^{42}$ and the temperature was chosen such that the reduced temperature $\left(T_{r}=T / T_{C}^{L L}\right)$ was approximately $0.96-0.97$. The reasons for this choice of reduced temperature are many-fold. First and foremost, we have performed free energy surface calculations for the original Jagla potential $\left(r_{1} / r_{0}=1.72\right)$ at the same reduced temperatures (refer to Figure 6). Furthermore, this reduced temperature is well outside of the range of the reported ${ }^{42}$ uncertainties associated with the critical temperatures, while still maintaining high enough temperatures to ensure the fastest-possible dynamics. After determining the temperature for a particular parameterization, the approximate coexistence pressure was then estimated from the reported ${ }^{42}$ slope of the coexistence line for that $r_{1} / r_{0}$ value. Multiple pressures were investigated to account for the inherent uncertainty in the aforementioned estimation of the coexistence pressure, and reweighting in pressure was applied to the free energy surfaces search for a LLPT in the vicinity of the simulated state points. Free energy surfaces for $r_{1} / r_{0}=1.64$ are provided in Figure 15. For this parameterization, the LLPT still clearly exists, and we see the free energy barrier between the liquid phases is very comparable to those observed for the original Jagla parameterization with a system of the same size.

Referring to Figure 16, one sees that upon decreasing $r_{1} / r_{0}$ to 1.62 the qualitative features of the free energy surface begin to exhibit noticeable changes with the incursion of a distinctive trough at lower densities which appears to encroach upon the LDL basin. While higher $Q_{6}$ values were not systematically studied, it would appear that this trough leads to the stable crystal basin. We note that the appearance of an LDL basin in Figure 16 (b) is tenuous, as reweighting to $0.1132 \varepsilon_{0} / r_{0}^{3}$ from $0.115 \varepsilon_{0} / r_{0}^{3}$ indicates existence of an LDL basin, whereas reweighting to $0.1132 \varepsilon_{0} / r_{0}^{3}$ from $0.110 \varepsilon_{0} / r_{0}^{3}$ does not show a distinct $\left(\geq 1 k_{B} T\right)$ barrier between the LDL and the trough. At this point, additional simulations in the vicinity of $0.1132 \varepsilon_{0} / r_{0}^{3}$ are required. However, 


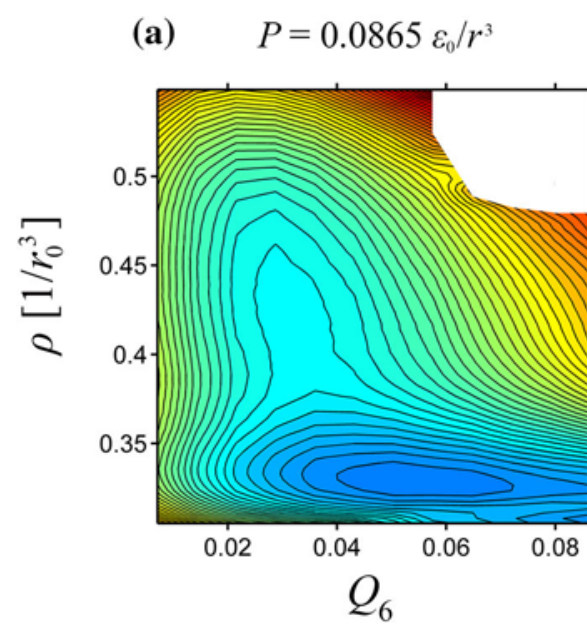

(b) $\quad P=0.0885 \varepsilon_{0} / r^{3}$

(c) $\quad P=0.0915 \varepsilon_{0} / r^{3}$

Figure 15. Free energy surfaces for the adjusted Jagla potential, with $r_{1} / r_{0}=1.64$. The simulation conditions are: $T=0.0614 \varepsilon_{0} / k_{B}, N=300$ particles, and pressures of (a) $0.0865 \varepsilon_{0} / r_{0}^{3}$, (b) $0.0885 \varepsilon_{0} / r_{0}^{3}$, and (c) $0.0915 \varepsilon_{0} / r_{0}^{3}$. The free energy surfaces were calculated by reweighting from a simulation performed at $P=0.0880 \varepsilon_{0} / r_{0}^{3}$ (not shown). Contour spacing is $1 k_{B} T$, and the statistical uncertainties are less than or approximately equal to $0.25 k_{B} T$ for most of the bins.

(a)

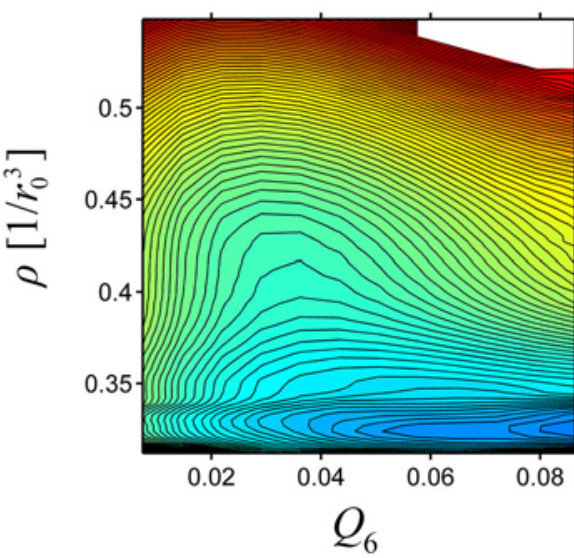

(b)

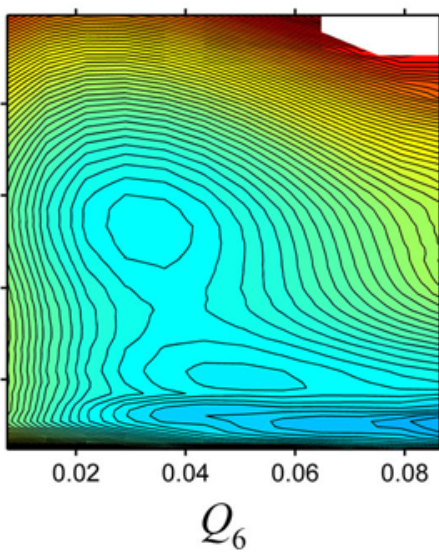

(c)

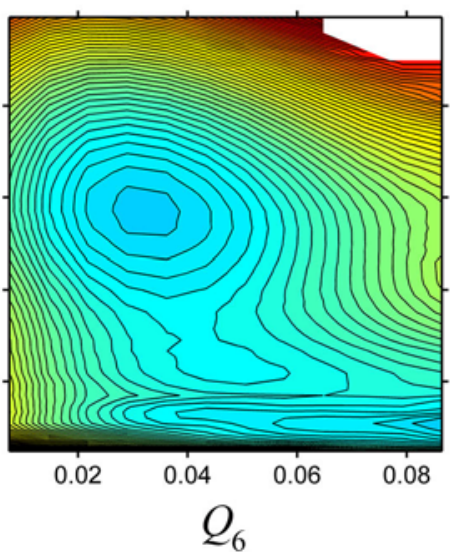

Figure 16. Free energy surfaces for the adjusted Jagla potential, with $r_{1} / r_{0}=1.62$. The simulation conditions are: $T=0.0618 \varepsilon_{0} / k_{B}, N=300$ particles, and pressures of (a) $0.110 \varepsilon_{0} / r_{0}^{3}$, (b) $0.1132 \varepsilon_{0} / r_{0}^{3}$, and (c) $0.115 \varepsilon_{0} / r_{0}^{3}$. The free energy surface in panel (b) was calculated by reweighting the surface shown in panel (c). Contour spacing is $1 k_{B} T$, and the statistical uncertainties are less than or approximately equal to $0.25 k_{B} T$ for most of the bins, except in the vicinity of the trough at low densities, where the uncertainty is on the order of $1-2 k_{B} T$.

regardless of whether or not a distinct barrier exists between the LDL and the steep trough at these conditions, it is clear that the signature of an LDL basin is weak at best.

When the $r_{1} / r_{0}$ ratio is further lowered to 1.60 , as shown in Figure 17, no evidence of an LDL basin is found for the range of temperatures and pressures explored. Indeed, only a single liquid basin appears in the free energy surface, along with an even more pronounced trough. Reweighting between pressures 1.40 and $1.45 \varepsilon_{0} / r_{0}^{3}$, one observes that the depth of the sin- gle, high-density, liquid basin decreases with decreasing pressure, as expected, until the liquid becomes unstable. We did not find any evidence of an LDL basin at these conditions for $r_{1} / r_{0}=1.60$.

We also note that, unlike the state points explored for $r_{1} / r_{0}=1.62$ and 1.64, the autocorrelation functions of density (and to a lesser extent, $Q_{6}$ ) for $r_{1} / r_{0}=1.60$ exhibit extreme dynamic heterogeneity in the low density region (i.e., in the vicinity of the trough shown in Figure 17) at the conditions explored. This behavior is illustrated in Figure 18. At present, it remains unclear 


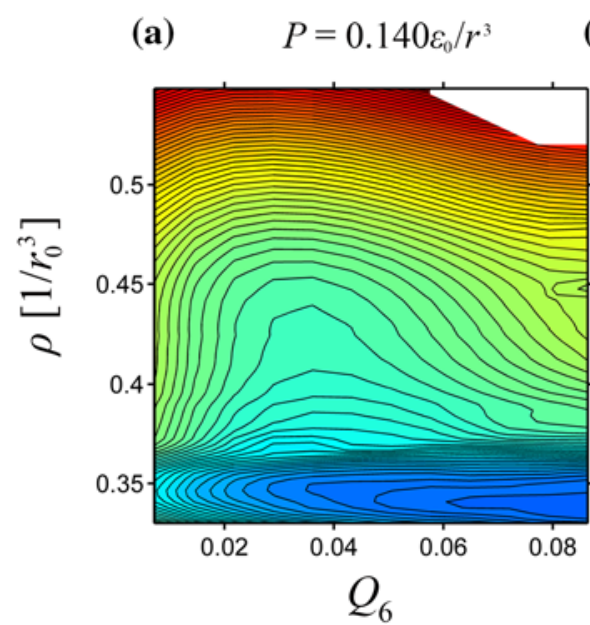

(b)

$P=0.1415 \varepsilon_{0} / r^{3}$

(c) $P=0.145 \varepsilon_{0} / r^{3}$
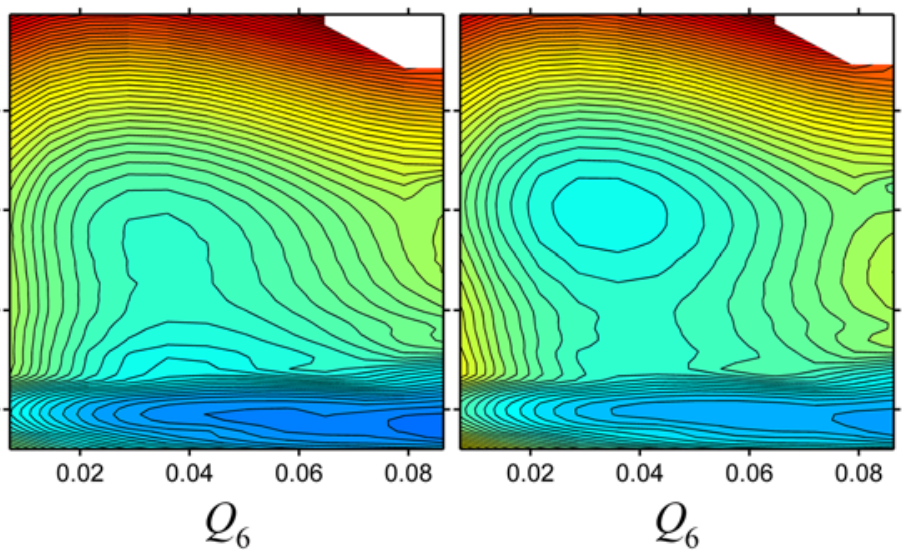

Figure 17. Free energy surfaces for the adjusted Jagla potential, with $r_{1} / r_{0}=1.60$. The simulation conditions are: $T=0.0616 \varepsilon_{0} / k_{B}, N=300$ particles, and pressures of (a) $0.140 \varepsilon_{0} / r_{0}^{3}$, (b) $0.1415 \varepsilon_{0} / r_{0}^{3}$, and (c) $0.145 \varepsilon_{0} / r_{0}^{3}$. The free energy surface in panel (b) was calculated by reweighting the surface shown in panel (c). Contour spacing is $1.5 k_{B} T$ in order to more clearly visualize the trough region, and the statistical uncertainties are less than or approximately equal to $0.25 k_{B} T$ for most of the bins, except in the vicinity of the trough at low densities, where the uncertainty is on the order of $1-2 k_{B} T$.
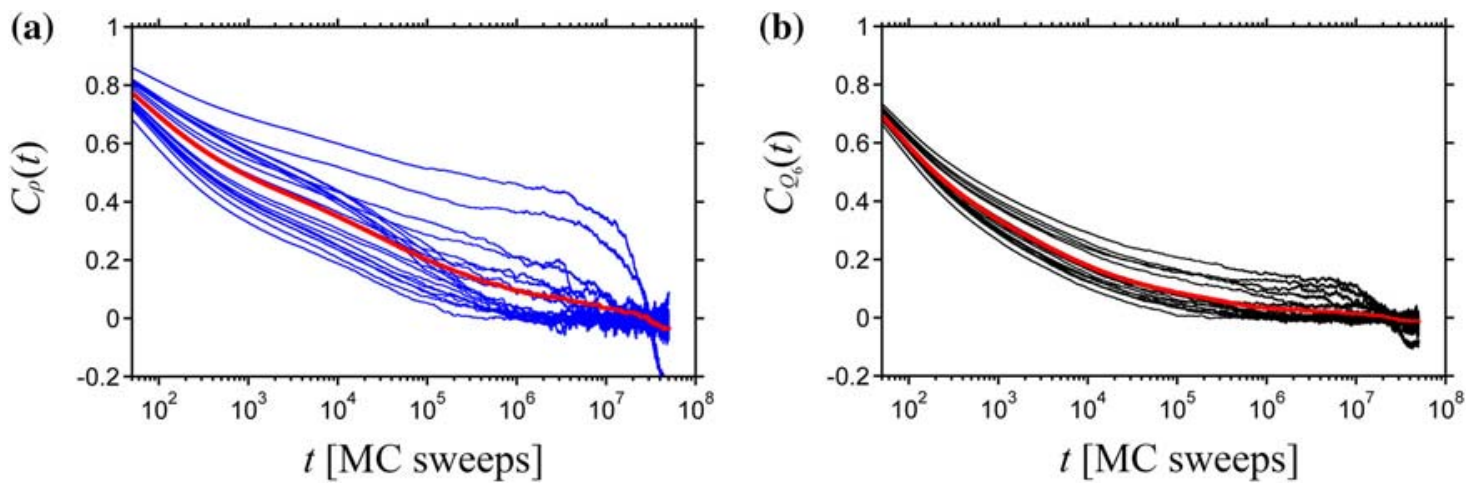

Figure 18. Autocorrelation functions of (a) density and (b) $Q_{6}$ for the adjusted Jagla potential, with $r_{1} / r_{0}=1.60$. The simulations are NPT MC subject to umbrella constraints as described in Section 2.2. The simulation conditions are: $T=0.0616 \varepsilon_{0} / k_{B}$, and $P=0.145 \varepsilon_{0} / r_{0}^{3}(N=300$ particles $)$. The target values of density and $Q_{6}$ for the umbrella constraints are $\rho^{*}=0.035 r_{0}^{-3}$, and $Q_{6}^{*}=0.05$ in order to explore conditions in the vicinity of the low-density trough. The thin curves shown are the autocorrelation functions for each of the 20 independent simulations, whereas the thick red curves are the average autocorrelation functions of all 20 simulations. Notice the large degree of dynamic heterogeneity in density autocorrealtions.

as to why this markedly different behavior occurs; however, it is clear that such extreme dynamic heterogeneity indicates that proper equilibration at these conditions is very difficult.

In summary, as the length-scale ratio $r_{1} / r_{0}$ is lowered from the original Jagla parameterization (1.72) towards the Lennard-Jones limit, our free energy calculations indicate that the LLPT ceases to exist in the vicinity of $r_{1} / r_{0}=1.60$. This is coincidentally the region of parameter space where previous work reported ${ }^{42,50}$ that the LLCP was prohibitively difficult to locate due to the onset of spontaneous crystallization. This length-scale ratio also happens to be in the immediate vicinity of where the slope of the coexistence line was predicted to change from positive to negative. Lower temperatures and additional pressures will need to be explored in order to assure that the LDL phase does not exist for this range of parameterization, as the previously reported ${ }^{42}$ locus of liquid-liquid critical points may be inaccurate in this region. However, at the conditions we explored for $r_{1} / r_{0}=1.60$, we find that the autocorrelations of density and $Q_{6}$ begin to exhibit extreme dynamic heterogeneity at low densities, which makes accurate calculation of the free energy surface significantly more challenging. At this time, the reason for this change in relaxation behavior remains unclear. Interest- 
ing areas for future work include a detailed free energy study at additional parameterizations and state points, a detailed investigation into why a steep trough appears to form in the free energy surface as $r_{1} / r_{0}$ is decreased, and a determination of why dynamic heterogeneity of density autocorrelations becomes so pronounced in the low density region as $r_{1} / r_{0}$ is decreased.

\section{A.4 The relationship between the Landau free energy and the thermodynamic free energy of phases}

We take this opportunity to clarify a point which has hitherto not been explicitly discussed in several works regarding free energy surface studies of liquid-liquid phase transitions. The free energy described by Equation (16) is a Landau free energy, also referred to as a free energy density, or a potential of mean force, which is an abstraction of the free energy over a range of order parameter space. ${ }^{57,75}$ Since we perform our calculations in the NPT ensemble, application of Equation (16) yields an abstraction of the Gibbs free energy. In order to obtain the Gibbs free energy associated with a particular phase at a given temperature and pressure, $G(T, P)$, one must perform a double-integration of the surface over both order parameters, ${ }^{75}$ choosing the domains of integration based on the range of order parameters characterizing the phase of interest. The resulting value of the Gibbs free energy is insensitive to the precise values of the domain of integration, so long as the region containing the local minimum in the free energy surface is included. ${ }^{57,75}$ We also note that, as mentioned elsewhere ${ }^{57}$ when comparing multiple free energy basins on a single surface (i.e., HDL and LDL basins) equality of basin depths implies equality of Gibbs free energies, unless the curvatures of the two basins are very different. This has been confirmed in our work, where for a given temperature, the equality of HDL and LDL basin depths is achieved at the equilibrium pressure predicted independently from equation of state calculations via Maxwell equal-area construction.

\section{References}

1. Poole P H, Sciortino F, Essmann U and Stanley H E 1992 Phase-behavior of metastable water Nature $\mathbf{3 6 0} 324$

2. Mishima O and Stanley H E 1996 Decompressioninduced melting of ice IV and the liquid-liquid transition in water Nature 392164

3. Liu Y, Panagiotopoulos A Z and Debenedetti P G 2009 Low-temperature fluid-phase behavior of ST2 water $J$. Chem. Phys. 1311045085

4. Limmer D T and Chandler D 2011 The putative liquidliquid transition is a liquid-solid transition in atomistic models of water J. Chem. Phys. 135134503
5. Liu Y, Palmer J C, Panagiotopoulos A Z and Debenedetti P G 2012 Liquid-liquid transition in ST2 water J. Chem. Phys. 137214505

6. Limmer D T and Chandler D 2013 The putative liquidliquid transition is a liquid-solid transition in atomistic models of water II J. Chem. Phys. 138214504

7. Palmer J C, Car R and Debenedetti P G 2013 The liquidliquid transition in supercooled ST2 water: a comparison between umbrella sampling and well-tempered metadynamics Faraday Discuss. 16777

8. Poole P H, Bowles R K, Saika-Voivod I and Sciortino F 2013 Free energy surface of ST2 water near the liquidliquid phase transition J. Chem. Phys. 138034505

9. Palmer J C, Martelli F, Liu Y, Car R, Panagiotopoulos A Z and Debenedetti P G 2014 Metastable liquid-liquid transition in a molecular model of water Nature $\mathbf{5 1 0} 385$

10. Chandler D 2014 Illusions of phase coexistence: Comments on "Metastable liquid-liquid transition" by J C Palmer et al. Nature 510385 arXiv:1407.6854v2

11. Palmer J C, Debenedetti P G, Car R and Panagiotopoulos A Z 2014 Response to Comment (arXiv:1407.6854) on Palmer et al. Nature 510385 (arXiv:1407.6854)

12. Binder K 2014 Simulations clarify when supercooled water freezes into glassy structures Proc. Natl. Acad. Sci. USA 1119374

13. Yagasaki T, Matsumoto $\mathrm{M}$ and Tanaka H 2014 Spontaneous liquid-liquid phase separation of water Phys. Rev. E 89020301

14. Limmer D T and Chandler D 2015 Comment on "Spontaneous liquid-liquid phase separation of water" Phys. Rev. E 91016301

15. Yagasaki T, Matsumoto M and Tanaka H 2015 Reply to Comment on "Spontaneous liquid-liquid phase separation of water" Phys. Rev. E 91016302

16. Limmer D T and Chandler D 2015 Time scales of supercooled water and implications for reversible polyamorphism Mol. Phys. 1131

17. Smallenburg F and Sciortino F 2015 Tuning the liquidliquid transition by modulating the hydrogen-bond angular flexibility in a model of water Phys. Rev. Lett. 115 015701

18. Speedy R J 1982 Stability-limit conjecture. An interpretation of the properties of water J. Phys. Chem. 86 982

19. Sastry S, Debenedetti P G, Sciortino F and Stanley H E 1996 Singularity-free interpretation of the thermodynamics of supercooled water Phys. Rev. E 536144

20. Debenedetti P G 2003 Supercooled and glassy water $J$. Phys.: Condens. Matter 15 R1669

21. Mishima O, Calvert L D and Whalley E 1984 Melting Ice-I at 77-K and 10-kbar - A new method of making amorphous solids Nature $\mathbf{3 1 0} 393$

22. Mishima O, Calvert L D and Whalley E 1985 An apparently 1st-order transition between 2 amorphous phases of ice induced by pressure Nature $\mathbf{3 1 4} 76$

23. Aptekar L I 1979 Phase transitions in noncrystalline germanium and silicon Sov. Phys. Dokl. 24933

24. Sastry S and Angell C A 2003 Liquid-liquid phase transition in supercooled silicon Nature Mater. 2739

25. Ganesh P and Widom M 2009 Liquid-liquid transition in supercooled silicon determined by first-principles simulation Phys. Rev. Lett. 102075701 
26. Beye M, Sorgenfrei F, Schlotter W F, Wurth W and Fohlisch A 2010 The liquid-liquid phase transition in silicon revealed by snapshots of valence electrons Proc. Natl. Acad. Sci.USA 10716772

27. Vasisht V V, Saw S and Sastry S 2011 Liquid-liquid critical point in supercooled silicon Nature Phys. 7549

28. Ricci F, Palmer J C and Debenedetti P G A computational investigation of the thermodynamics of the StillingerWeber family of models at supercooled conditions in preparation

29. Poole P H, Hemmati M and Angell C A 1997 Comparison of thermodynamic properties of simulated liquid silica and water Phys. Rev. Lett. 792281

30. Saika-Voivod I, Sciortino F and Poole P H 2000 Computer simulations of liquid silica: Equation of state and liquid-liquid phase transition Phys. Rev. E 631

31. Lascaris E, Hemmati M, Buldyrev S V, Stanley H E and Angell C A 2014 Search for a liquid-liquid critical point in models of silica J. Chem. Phys. 140224502

32. Mishima O 2000 Liquid-liquid critical point in heavy water Phys. Rev. Lett. 85334

33. Stillinger F H and Rahman A 1974 Improved simulation of liquid water by molecular dynamics J. Chem. Phys. 60 1545

34. Stillinger F H and Weber T A 1985 Computer simulation of local order in condensed phases of silicon Phys. Rev. B 315262

35. Molinero V and Moore E B 2009 Water modeled as an intermediate element between carbon and silicon J. Phys. Chem. B 1134008

36. Smallenburg F, Filion L and Sciortino F 2014 Erasing noman's land by thermodynamically stabilizing the liquidliquid transition in tetrahedral particles Nature Phys.10 653

37. Hemmer P C and Stell G 1970 Fluids with several phase transitions Phys. Rev. Lett 241284

38. Stell G and Hemmer P C 1972 Phase-transitions due to softness of potential core J. Chem. Phys. 564274

39. Mishima O and Stanley H E 1998 The relationship between liquid supercooled and glassy water Nature 396 329

40. Jagla E A 2001 Liquid-liquid equilibrium for monodispersesphericalparticles Phys. Rev. E 63061501

41. Wilding N B and Magee J E 2002 Phase behavior and thermodynamic anomalies of core-softened fluids Phys. Rev. E 66031509

42. Gibson H M and Wilding N B 2006 Metastable liquidliquid coexistence and density anomalies in a coresoftened fluid Phys. Rev. E 73061507

43. Xu L M, Buldyrev S V, Angell C A and Stanley H E 2006 Thermodynamics and dynamics of the two-scale spherically symmetric Jagla ramp model of anomalous liquids Phys. Rev. E 74031108

44. Errington J R and Debenedetti P G 2001 Relationship between structural order and the anomalies of liquid water Nature 409318

45. Angell C A 1993 Water-II is a "strong" liquid J. Chem. Phys. 976339

46. Xu L M, Kumar P, Buldyrev S V, Chen S H, Poole P H, Sciortino F and Stanley H E 2005 Relation between the Widom line and the dynamic crossover in systems with a liquid-liquid phase transition Proc. Natl. Acad. Sci. USA 10216558

47. Xu L M, Giovambattista N, Buldyrev S V, Debenedetti P G and Stanley H E 2011 Waterlike glass polyamorphism in a monoatomic isotropic Jagla model J. Chem. Phys. 134064507

48. Abraham J Y, Buldyrev S V and Giovambattista N 2011 Liquid and glass polymorphism in a monatomic system with isotropic smooth pair interactions J. Phys. Chem. B 11514229

49. Yan Z Y, Buldyrev S V, Kumar P, Giovambattista N and Stanley H E 2008 Correspondence between phase diagrams of the TIP5P water model and a spherically symmetric repulsive ramp potential with two characteristic length scales Phys. Rev. E 77042201

50. Luo J Y, Xu L M, Angell C A, Stanley H E and Buldyrev S V 2015 Physics of the Jagla model as the liquid-liquid coexistence line slope varies J. Chem. Phys. 142224501

51. Yan Z Y, Buldyrev S V, Giovambattista N, Debenedetti P G and Stanley H E 2006 Family of tunable spherically symmetric potentials that span the range from hard spheres to waterlike behavior Phys. Rev. E 73051204

52. Buldyrev S V, Malescio G, Angell C A, Giovambattista N, Prestipino S, Saija F, Stanley H E and Xu L 2009 Unusual phase behavior of one-component systems with two-scale isotropic interactions J. Phys.: Condens. Matter 21504106

53. $\mathrm{Xu} \mathrm{L} \mathrm{M,} \mathrm{Buldyrev} \mathrm{S} \mathrm{V,} \mathrm{Giovambattista} \mathrm{N,} \mathrm{Angell} \mathrm{C}$ A and Stanley H E 2009 A monatomic system with a liquid-liquid critical point and two distinct glassy states J. Chem. Phys. 130054505

54. Buldyrev S V, Kumar P, Sastry S, Stanley H E and Weiner S 2010 Hydrophobic collapse and cold denaturation in the Jagla model of water J. Phys. Condens. Matter 22 284109

55. de Miguel E and Jackson G 2006 The nature of the calculation of the pressure in molecular simulations of continuous models from volume perturbations J. Chem. Phys. 125164109

56. Steinhardt P J, Nelson D R and Ronchetti M 1983 Bondorientational order in liquids and glasses Phys. Rev. B 28 784

57. Lynden-Bell R M, van Duijneveldt J S and Frenkel D 1993 Free-energy changes on freezing and melting ductile metals Mol. Phys. 80801

58. Truskett T M, Torquato S and Debenedetti P G 2000 Towards a quantification of disorder in materials: Distinguishing equilibrium and glassy sphere packings Phys. Rev. E 62993

59. Mountain R D and Raveche H J 1971 Entropy and molecular correlation functions in open systems II Two-body and three-body correlations J. Chem. Phys. 552250

60. Baranyai A and Evans D J 1989 Direct entropy calculation from computer-simulation of liquids Phys. Rev. A $\mathbf{4 0}$ 3817

61. Kumar S, Bouzida D, Swendsen R H, Kollman P A and Rosenberg J M 1992 The weighted histogram analysis method for free-energy calculations on biomolecules 1 . The method J. Comput. Chem. 131011

62. Hub J S, de Groot B L and van der Spoel D 2010 g_whamA free weighted histogram analysis implementation 
including robust error and autocorrelation estimates $J$. Chem. Theory Comput. 63713

63. Allen M P and Tildesley D J 1987 Computer Simulation of Liquids (New York: Oxford University Press)

64. Shirts M R and Chodera J D 2008 Statistically optimal analysis of samples from multiple equilibrium states $J$. Chem. Phys. 129124105

65. Yashonath S and Rao C N R 1985 A Monte Carlo study of crystal structure transformations Mol. Phys. 54245

66. Palmer J C, Singh R S, Chen R, Martelli F and Debenedetti P G 2016 Density and bond-orientational relaxations in supercooled water Mol. Phys. 1142580

67. Errington J R 2003 Evaluating surface tension using grand-canonical transition-matrix Monte Carlo simulation and finite-size scaling Phys. Rev. E 67012102

68. Binder K 1982 Monte Carlo calculation of the surface tension for two- and three-dimensional lattice-gas models Phys. Rev. A 251699

69. Hunter J E and Reinhardt W P 1995 Finite-size scaling behavior of the free energy barrier between coexisting phases: Determination of the critical temperature and interfacial tension of the Lennard-Jones fluid $J$. Chem. Phys. 1038627

70. Berg B A, Hansmann U and Neuhaus T 1993 Properties of interfaces in the two and three dimensional Ising model Z. Phys. B 90229

71. Widom B 1965 Surface tension and molecular correlations near the critical point J. Chem. Phys. 433892

72. Gallo P and Sciortino F 2012 Ising universality class for the liquid-liquid critical point of a one component fluid: A finite-size scaling test Phys. Rev. Lett. 109177801

73. van Duijneveldt J S and Frenkel D 1992 Computer simulation study of free energy barriers in crystal nucleation J. Chem. Phys. 964655

74. Frenkel D and Smit B 2002 Understanding Molecular Simulation: From Algorithms to Applications $2^{\text {nd }}$ ed. (USA: Academic Press)

75. Radhakrishnan R and Trout B L 2005 Order parameter approach to understanding and quantifying the physicochemical behavior of complex systems In Handbook of Materials Modeling S Yip (Ed.) (Dordrecht Netherlands: Kluwer Academic Publishers) pp. 1613-1626 